\title{
Mechanics of Shock Induced Pore Collapse in Poly(methyl methacrylate) (PMMA): Comparison of Simulations and Experiments
}

\author{
Nirmal Kumar Rai ${ }^{1}$, Emilio M. Escauriza ${ }^{2}$, Daniel E. Eakins ${ }^{2}$ and H.S. Udaykumar ${ }^{1}$ \\ ${ }^{1}$ Department of Mechanical Engineering, The University of Iowa, Iowa City, IA-52242 \\ ${ }^{2}$ Department of Engineering Science, University of Oxford, Oxford, OX1 3PJ, UK
}

\begin{abstract}
Head-to-head comparisons are made between calculations and experimental data on shock-driven pore collapse in the transparent material, poly(methyl methacrylate) (PMMA). Simulations are performed using SCIMITAR3D, an Eulerian sharp-interface multi-material code, while plate impact experiments are visualized using ultra-high speed x-ray imaging. The experiments and simulations are conducted over a wide range of loading conditions; from low strength loading regimes where adiabatic shear banding predominates all the way up to the regime where hydrodynamic pore collapse is expected. PMMA is modeled using an isotropic rate-dependent plasticity model for the deviatoric stress response and a Tillotson equation of state for the pressure. Calculations are primarily done in 2D, to save computational effort, but a limited number of 3D calculations are also performed to assess the differences entailed by the dimensionality. The 2D calculations are in fairly good agreement with the experimental results, for the evolution of pore shape. 3D calculations, while quite computationally intense, indeed produce better agreement with experimental data. The computations also agree well with the experiments in delineating the loading strength at which a transition from the strength-dominated to hydrodynamics-dominated pore collapse occurs. This work provides confidence in the ability of Eulerian, sharp interface computational techniques to correctly represent and understand the mechanics of shock-loaded porous condensed phase materials over a range of loading conditions.
\end{abstract}

Keywords: Pore collapse, cavity collapse, void collapse, PMMA, energetic materials, adiabatic shear bands, shocks, rate dependent plasticity, hydrodynamic collapse, jetting

\section{Introduction}

Pore collapse is an important mechanism for energy localization in heterogeneous materials. In liquids this localization results in jet and vortex formation, which has been harnessed for applications in the medical sciences, such as targeted drug delivery $[1,2]$ and the destruction of kidney and gall stones through shock lithotripsy [3, 4] among others. In solid media, which is the focus of the present work, the energy localization can be more complex, and has primarily been studied in relation to heterogenous energetic materials (HEs). Pore collapse in HEs leads to energy localization at hotspots primarily at high loading pressures, i.e. when the applied shock leads to pressures considerably exceeding the yield strength of the material. Closer to the yield stress and certainly below it, other mechanisms of energy localization may play important roles. These may include adiabatic shear bands, plastic dissipation, friction etc. [5]. Numerical simulations must therefore accurately capture the variety of possible energy localization mechanisms. In this work, we examine the mechanics of shock driven pore collapse in a solid inert polymer material poly(methyl methacrylate) (PMMA) under loading regimes where shear bands and jetting are the primary modes of energy localization.

It is well known that pore collapse in HEs leads to intense hotspots that can grow into sustained reactive fronts that consume the surrounding solid material. In the case of a single pore [6-13] or a field of pores $[11,12,14,15]$, the thermo-mechanics of pore collapse has now been well studied using simulations. 
Benchmarking has been done to some extent $[9,16]$ and issues such as the resolution requirements to obtain reliable calculations of hotspot temperatures have been investigated [9]. However, even for primarily hydrodynamic pore collapse, and even in well-studied materials such as HMX and TATB, comparison of numerical calculations against experimental data for wide range of loading conditions has not yet been achieved. Recent work by Durate et al. [17] has shown good agreement with experimental data for pore collapse in HMX in the weak shock loading regime, i.e. particle speed of $0.1 \mathrm{~km} / \mathrm{s}$. Some preliminary, qualitative comparisons for pore collapse in HMX were also shown in Rai et al. [9] against the experimental pore collapse profiles of Swantek and Austin [11]. However, quantitative head-to-head comparison of calculated pore collapse behavior and temperatures for wide range of loading scenarios against experiments is still lacking, which is a significant gap in the literature on hotspot formation in HEs. Establishing the accuracy of numerical simulations, particularly as they relate to hotspot ignition and growth, is crucial to enhancing our ability to model and predict the sensitivity of shock-loaded HEs.

The situation regarding paucity of quantitative comparison of models and experiments is not markedly better for other regimes of pore collapse. For example, when the shock strength is lowered from pressures of several GPa (flyer impact velocities of $\mathrm{km} / \mathrm{s}$ ) to hundreds of MPa (flyer impact velocities of $100 \mathrm{~m} / \mathrm{s}$ ), the importance of inertia is diminished. Previous pore collapse studies in HMX $[6,10,18]$ have suggested there can be considerable energy localization in shear bands emanating from pore surfaces as the shock strength is lowered. These studies capture the localized plastic dissipation in the adiabatic shear bands using plasticity models that vary in sophistication from semi-empirical isotropic rate dependent models for HMX $[6,18]$ to sophisticated - but computationally intensive - calculations using anisotropic crystal plasticity treatments [10]. Both types of calculations produce physically realistic shear band profiles, irrespective of the complexity of the material model involved. Rate-dependent plasticity effects appear sufficient to obtain shear bands exhibiting significant temperature excursions (with maximum temperature approaching the melting point). The shock intensity which marks the transition from inertia-dominated to shear-band dominated energy localization is not precisely established in these models; the transition point is material dependent. In addition, while some interesting simulations of the shear banding phenomena have been shown $[6,10,18]$, no direct comparison with experiments in any energetic material has yet been performed.

The present work addresses the above knowledge gaps by comparing numerical simulations of pore collapse directly against sub-surface X-ray imaging experiments; the head-to-head comparison is performed over a range of loading conditions spanning shock strengths for which shear bands predominate to those firmly in the hydrodynamic range. A sharp interface Eulerian level-set based approach [19-21] is used to calculate the dynamics of a single pore embedded in a solid material matrix. The geometry used in the simulations mimics that used in experiments, allowing for direct comparison of the deformation of pores at several time instants. The comparison is performed for the inert non-crystalline material PMMA, which facilitates visualization in experiments due to its relatively low atomic weight, resulting in low X-ray attenuation.

The paper is organized as follows. In Section 2, a brief summary of the numerical and experimental methods used in this work are presented. Section 3 presents the results of the pore collapse simulations and comparison with experiments over the period of pore deformation and collapse. 2D and 3D simulation results are compared to each other and to experiments to assess the error resulting from adopting the far more tractable 2D approximation. Section 4 summarizes the main conclusions of this work.

\section{Methods}

In this section, we present a summary of the numerical framework and experimental set-up used to analyze the collapse behavior of pores in PMMA. A more comprehensive discussion of the numerical 
framework is presented in previous work $[9,20]$. The details of the experimental method and set up are also available in a previous publication [22].

\subsection{Computational Framework}

The collapse of a pore in PMMA is simulated using a Cartesian grid Eulerian based sharp interface code, SCIMITAR3D [19, 20]. The governing equations in the Eulerian framework comprise the set of hyperbolic conservation laws. In Cartesian coordinates, we solve the governing equations of conservation of mass, momentum and energy:

$$
\begin{gathered}
\frac{\partial \rho}{\partial t}+\frac{\partial\left(\rho u_{i}\right)}{\partial x_{i}}=0 \\
\frac{\partial\left(\rho u_{i}\right)}{\partial t}+\frac{\partial\left(\rho u_{i} u_{j}-\sigma_{i j}\right)}{\partial x_{j}}=0
\end{gathered}
$$

and

$$
\frac{\partial(\rho E)}{\partial t}+\frac{\partial\left(\rho E u_{j}-\sigma_{i j} u_{i}\right)}{\partial x_{j}}=0
$$

where $\rho$, and $u_{i}$, respectively denote the density, and the velocity components, $E=e+\frac{1}{2} u_{i} u_{i}$ is the specific total energy, and $e$ is the specific internal energy. The Cauchy stress tensor, $\sigma_{i j}$ is of the form:

$$
\sigma_{i j}=S_{i j}-p \delta_{i j}
$$

where $S_{i j}$ is the deviatoric stress tensor and $p$ is the pressure.

In the current Prandlt Reuss [23] formulation, the deviatoric stress components, $S_{i j}$ are evolved using the following equations,

$$
\frac{\partial\left(\rho S_{i j}\right)}{\partial t}+\frac{\partial\left(\rho S_{i j} u_{k}\right)}{\partial x_{k}}+\rho S_{i k} \Omega_{k j}-\rho \Omega_{i k} S_{k j}=\rho G\left(D_{i j}^{d}-D_{i j}^{d, p}\right)
$$

where $D_{i j}^{d}$ is the deviatoric component of the strain rate tensor, $\Omega_{i j}$ is the spin tensor, $D_{i j}^{d, p}$ is the plastic component of the deviatoric strain rate tensor and $G$ is the shear modulus of the material. Using Eq. (5), the deviatoric stresses are evolved by the elastic update and then followed by mapping back to the yield surface using the radial return algorithm [24]. It is important to note that the viscoelastic behavior of PMMA is not modeled in the present work.

The yield surface for PMMA is defined using the isotropic rate dependent Johnson-Cook model given as,

$$
\sigma_{Y}=\left[A+B \varepsilon_{p}^{n}\right]\left[1+C \ln \left(\dot{\varepsilon}^{*}\right)\right]\left[1-T^{* m}\right]
$$

where, $\sigma_{Y}$ is the yield stress, $\varepsilon_{p}$ is the equivalent plastic strain, $\dot{\varepsilon}^{*}=\dot{\varepsilon} / \dot{\varepsilon}_{0}, \dot{\varepsilon}$ is the plastic strain rate, and $\dot{\varepsilon}_{0}$ is the dimensionless plastic strain rate. The homologous temperature, $T^{*}=\frac{T-T_{r e f}}{T_{M E L T}-T_{r e f}}, \mathrm{~T}$ is the temperature of the material, $T_{r e f}$, reference temperature and $\dot{\varepsilon}_{0}=1 s^{-1} . A, B, n, C$ and $m$ are Jonson Cook model parameters which are provide in Table I [25]. The isotropic rate dependent plastic behavior of PMMA is modeled until the temperature reaches the melting temperature of PMMA. After melting takes 
place, the deviatoric stress components, i.e. $S_{i j}$ is set to 0 and the mechanical behavior of PMMA is only governed by the pressure.

The pressure, $p$ for PMMA is provided by the Tillotson equation of state [26],

$$
p=\left[a+\frac{b}{\left(\frac{E}{\left(E_{0} \eta^{2}\right)}+1\right)}\right] \rho e+A \mu+B \mu^{2}
$$

where, $\eta=\rho / \rho_{0}, \mu=\eta-1$ and $a, b, A, B$ and $E_{0}$ are the Tillotson EOS parameters calibrated against the shock hugoniot data for PMMA presented in [27]. The EOS parameters are provided in Table 1. Eq. (7) is used to model the compression in PMMA under shock loading. In the expansion regime, i.e. $\eta>1$, the following pressure equation is used,

$$
p_{h}=a \rho e+\left[\frac{b \rho e}{\left(\frac{E}{\left(E_{0} \eta^{2}\right)}+1\right)}+A \mu e^{-\beta \mu}\right] e^{-\alpha \mu^{2}}
$$

where, $\alpha$ and $\beta$ are the constants and their values are provided in Table I.

The temperature rise in PMMA is computed from the internal energy as,

$$
T=T_{r e f}+\frac{e-e_{0}}{C_{v}}
$$

where, $T_{\text {ref }}=298 \mathrm{~K}$ is the reference temperature, $e_{0}$ is the reference energy on the cold curve at $298 \mathrm{~K}$ and $C_{v}$ is the specific heat at constant volume for PMMA at $298 \mathrm{~K}$ given in Table I.

In the present analysis, a constant value of $C_{v}$ is used to calculate the temperature because of lack of temperature and pressure dependent relation of $C_{v}$ for PMMA. The constant $C_{v}$ assumption can lead to the overestimation of temperature. As a crude estimation, the upper and lower bound of $C_{v}$ can be used to obtain the bounds on the temperature rise from the pore collapse in PMMA. The lower bound of $C_{v}$ at 298 $\mathrm{K}$ is used in the current analysis, i.e. $C_{v}^{298 K}=1466 \mathrm{~J} / \mathrm{Kg} \mathrm{K}$ and the classical limit (CL) of $C_{v}$ given by Dulong-Petit law [28] defines the upper bound of $C_{v}$, i.e. $C_{v}^{C L}=3741.3 \mathrm{~J} / \mathrm{Kg} \mathrm{K}$.

The upper bound of temperature, i.e. $T_{C_{v}^{298 K}}$ is obtained using Eq. (9) with $C_{v}^{298 K}$. On the other hand, the lower bound of temperature is given by $T_{C_{v}^{C L}} \cdot T_{C_{v}^{C L}}$ is related to $T_{C_{v}^{298 K}}$ as, $T_{C_{v}^{C L}}=T_{r e f}+$ $\frac{C_{v}^{298 K}}{C_{v}^{C L}}\left(T_{C_{v}^{298 K}}-T_{\text {ref }}\right)$. The temperature data presented in the results section is given by $T_{C_{v}^{298 K}}$, however, it is important to note that in reality the temperature rise from the pore collapse is expected to vary between $T_{C_{v}^{C L}}$ and $T_{C_{v}^{298 K} \text {. }}$

\subsection{Numerical Algorithms and Interfacial Conditions}

The conservation laws of mass, momentum and energy (Eqs. (1-3)), along with the evolution of deviatoric stresses (Eq. 5) are spatially discretized using a $3^{\text {rd }}$ order shock capturing ENO scheme [29]. A $3^{\text {rd }}$ order Runge-Kutta scheme is used for the temporal discretization. The pore interface is represented using a narrow-band levelset based sharp interface method [30]. The free surface conditions between the PMMA and pore interface are applied using a modified ghost fluid method [31]. A more comprehensive 
descriptions of the spatial and temporal discretization schemes, narrow-band level set implementation and ghost fluid method are provided in previous works [19-21, 32].

\subsection{Experimental Setup}

In this section, a brief description of the experimental and imaging set-up used to analyze the shock induced collapse behavior of spherical pores in PMMA is presented. Detailed overview of the experimental methods is presented in the work by Escauriza et al. [22].

The experiments were performed at beamline ID19 of the European Synchrotron Radiation Facility (ESRF), where both a single-stage and a two-stage gas gun were used to dynamically compress targets containing a single spherical pore under a wide range of shock loading conditions. Figure 1 shows the experimental set-up used to analyze the behavior of the pores under shocks. The targets were constructed from PMMA, consisted of two cylindrical sections glued together - see Figure 1(b). Both sections had a hemispherical portion removed from one end, which created a spherical pore inside a single cylinder when joined. The central orientation of the pore within the targets created an axially symmetric design. Nine experiments were performed on the single-stage gas gun, which launched projectiles constructed from a sabot and flyer plate. The targets for these impacts contained pores with diameters 4 and $6 \mathrm{~mm}$. For seven of the experiments, an aluminum (2024 alloy) flyer was used, generating initial particle velocities behind the shock front from 0.16 to $0.59 \mathrm{~km} / \mathrm{s}$. For two of the experiments, the pore target was mounted on the sabot as a flyer and impacted against a large steel anvil, generating particle velocities of 0.65 and $0.71 \mathrm{~km} / \mathrm{s}$. A further eight experiments were performed on the two-stage gas gun, for which the sabot diameter was $10 \mathrm{~mm}$. Polycarbonate flyer plates with diameter $9 \mathrm{~mm}$ were impacted against targets with pores of diameter 3,4 and $6 \mathrm{~mm}$, generating particle velocities between 0.99 and $2.3 \mathrm{~km} / \mathrm{s}$.

With reference to Figure 1(a), which shows the configurations for the experimental and imaging systems: to record the pore deformation under applied loading, a custom indirect ultra-high-speed (UHS) $\mathrm{x}$-ray imaging (XRI) system design was used, the full details of which were given by Escauriza et al. [22]. UHS is defined here as $>1$ million frames per second (fps). The imaging system configuration is also shown in Figure 1(a). The radiographs were recorded 'indirectly' using visible light cameras, by first absorbing the $x$-ray photons with a scintillator. The scintillator re-emits visible photons from its front and rear surfaces. Both visible light paths were folded away from the $\mathrm{x}$-ray beam path with pellicle beam splitters, located up-range and down-range of the scintillator, toward two Shimadzu Hypervision HPV-X2 cameras. For the single-stage experiments, one of the cameras was used, which recorded an image every third X-ray pulse, thus giving an inter-frame time of $528 \mathrm{~ns}$ and a frame rate of $1.89 \mathrm{Mfps}$. Both cameras were used for the two-stage experiments and the radiographs were interleaved, capturing two-thirds of the x-ray pulses and producing radiograph sequences with effective inter-frame times of 176/352 ns and frame rates of 5.68/2.84 Mfps. A 1:1 magnification provided a field of view (FOV) approximately equal to the camera sensor size of $8 \mathrm{~mm} \times 12 \mathrm{~mm}$ (a $250 \times 400$ pixel array with a pixel size of $32 \times 32 \mu \mathrm{m}$ ).

For both the single-stage and two-stage setups, the end of the gas gun barrels was sealed inside a target chamber. Before firing, the barrel and target chamber were placed under vacuum to maximize the projectile velocity. At the end of the barrel, two light gates recorded the flyer velocity and were used to trigger the imaging system. The experiments were oriented such that the x-rays propagated perpendicular to the direction of the projectile and through the region of the target, providing a side-on radiographic projection of the impacts and pore collapse events. Two low-Z Mylar windows, one on each side of the target chamber, minimized the attenuation of the x-rays while still allowing a vacuum to be maintained. The ESRF's 16-bunch filling mode was utilized, in which a $100 \mathrm{ps}$ long x-ray pulse is produced every 176 ns [33]. The imaging system for recording the x-rays was placed $7 \mathrm{~m}$ from the experiment. Based on the system's specifications, this ensured that the radiographs were recorded in the phase contrast regime. 
Phase contrast increases the contrast of material edges and interfaces, thus improving the visibility of tracking details during the impacts, such as the dynamics of the pore interface and crack formation [34].

Using the experimental and imaging setups, the pore collapse behavior of the $3 \mathrm{~mm}, 4 \mathrm{~mm}$ and $6 \mathrm{~mm}$ diameter pores under low to high speed impact is analyzed. The results from the 17 experiments are presented in detail in the work of Escauriza et al. [22]. In the present work, the underlying physics governing the collapse behavior of a pore of given diameter under different loading strengths is studied. Therefore, the data for the $6 \mathrm{~mm}$ diameter pore under 4 different shock strengths are extracted from the experiments and compared against the simulation results. The details of the 4 experiments are presented in Table II.

\subsection{Extracting data from the images}

For a given experiment, the UHS x-ray imaging setup generates 2D radiographic high-resolution images of the deformed pore surface at different time instants. The images are $2 \mathrm{D}$ projections of the spherical pore target as can be seen from Figures 1(b) and 1(c). Interfaces in the images, such as the glue line, pore interface, and shock front, were enhanced by phase contrast interference fringes [35, 36]. Each radiograph was scaled by a 'flat-field' image taken prior to each experiment before the target was placed in the FOV; this process was necessary to flatten the intensity profile in the images and enhance edge detection.

Using the 2D radiographic images, quantitative data describing the pore collapse behavior are extracted by detecting the edges of the pore interface in the radiographs. Edge detection was achieved using the Canny method, which applies a Gaussian filter to an image before identifying edges as regions with a local gradient maximum [37]. Once the pore edge is detected, the projected pore area is computed using the number of pixels enclosed within the pore interface. The instantaneous deformed pore area is normalized with the initial projected area to measure the relative change in the pore area,

$$
A^{\prime}(t)=A(t) / A_{0}
$$

where, $A$ is the pore area at time $t$ and $A_{0}$ is the initial pore area. From the radiograph images at different time instants, the time to pore closure is calculated. The pore closure time is defined as time to reach minimum area, $t_{\mathrm{A}, \min }$ of a given pore under the applied shock, where zero time is referenced to the instant the shock first reaches the pore surface.

From the images, metrics describing the kinematic behavior of the pore collapse, including velocity and acceleration of the pore surface are also computed by measuring the displacement, $x$ of the pore interface along the pore centerline at different time instants. The centerline pore displacement is normalized by initial pore diameter to define the relative change in the centerline displacement with time. The dimensionless centerline pore displacement is, therefore,

$$
x^{\prime}(t)=x(t) / D_{0}
$$

where, $x$ is the centerline displacement at time $t$ and $D_{0}$ is the pore diameter before impact (in $\mathrm{mm}$ ). Similarly, the dimensionless time is given as,

$$
t^{\prime}=t C_{L} / D_{0}
$$

where $t$ is in $\mu s$ and $C_{L}$ is the longitudinal sound speed in PMMA.

In the study by Escauriza et al. [22], through simulations that included no strength model, it was established that in the hydrodynamic regime the pore centerline undergoes constant acceleration. In this 
regime the velocity and acceleration along the pore centerline are computed using a quadratic fit between the normalized centerline pore displacement, $x^{\prime}$ and dimensionless time, $t^{\prime}$ as,

$$
x^{\prime}(t)=a^{\prime} / 2 t^{\prime 2}+v_{0}^{\prime} t^{\prime}
$$

The coefficient $a^{\prime}$ of the fit defines the dimensionless centerline pore acceleration and $v_{0}^{\prime}$ is the dimensionless initial interface velocity, i.e. the release velocity of the mid-point of the pore interface. The dimensionless acceleration, $a^{\prime}$ is related to the acceleration, $a$, as,

$$
a^{\prime}=a D_{0} / C_{L}^{2}
$$

and the non-dimensionalized release velocity is defines as,

$$
v_{o}^{\prime}=\frac{v_{o}}{C_{L}}
$$

Also in the Escauriza et al. study [22], it was observed that for PMMA constant acceleration was identified for incident loading pressures $\geq 4.8 \mathrm{GPa}\left(U_{p} \geq 0.80 \mathrm{~km} / \mathrm{s}\right)$. However, for all the loading pressures studied, the magnitude of the acceleration was significantly less than that predicted by the hydrodynamic simulations, suggesting the persistence of effects from strength. This observation is supported by a Bat'kov et al. study [38] on the shock response of PMMA, which found non-vanishing deviatoric strength up to $15 \mathrm{GPa}$.

Using the quadratic fit, i.e. Eq. (13), the velocity along the pore interface is computed for the experiments in the hydrodynamic regime as,

$$
v^{\prime}(t)=a^{\prime} t^{\prime}+v_{0}^{\prime}
$$

where, $v^{\prime}(t)=v(t) / C_{L}$ is the non-dimensional pore centerline velocity. Using Eq. (16), the final velocity of the jet, $v_{j e t}^{\prime}$ is obtained when the upstream pore interface reaches the far pore interface along the centerline, and can be calculated by solving Eq. (13) with the best-fit $a^{\prime}$ and $v_{o}^{\prime}$ values for $x=D_{0}$. This gives the time at which the jet impacts the interface, $t^{\prime}\left(x=D_{0}\right)$, which is inserted into Eq. (16) to give

$$
v_{j e t}^{\prime}=\left.a^{\prime} t^{\prime}\right|_{x=d_{0}}+v_{0}^{\prime}
$$

\subsection{Methods for generating overlaid plots of pore deformation profiles obtained from simulations and experiments}

To perform a head-to-head visual comparison between the experimentally observed pore collapse behavior and numerical simulations, the deformed pore shapes at different time instants are extracted from the $2 \mathrm{D}$ radiographic images and are laid over the simulation pore shapes. A diagram demonstrating the steps taken to create the overlaid images can be seen in Figure 2. As can be seen in Figure 2(a), these images are first converted to a mask images through edge detection, with the pore area in white and the surrounding medium in black. The next step is to resize one of the images relative to the other through a two-dimensional affine transformation, necessary because of the different sizes of the experimental and simulation images. To obtain the transformation parameters, the mask images of the unshocked pore at $t=0 \mu \mathrm{s}$ are chosen, because at this time before impact both the data and simulation images have the same pore shape, i.e. projected circles. The registration algorithm attempts to find a transformation that will match features in the moving image (the image being transformed) to the fixed image. The result is two images with the same pixel dimensions, pore size and pore position. The obtained affine transformation parameters are then 
applied to all of the data images, producing a set of images for the data and simulations that are aligned and the same size. This is demonstrated in Figure 2(b), in which the top left data image is transformed to the top right image. Each data image is paired with a simulation image that has an interaction time in the same neighborhood $(0.840 \mu \mathrm{s}$ and $0.845 \mu \mathrm{s}$ in the Figure 2(b) example). The final overlaid image is produced by taking the edge of the simulation mask image and registered data mask image and placing them overlaid on a white background. In the example shown, black and red lines were chosen for the data and simulation images, respectively.

\section{Results and discussion}

In this study, head-to-head comparison between numerical simulations and experiments are performed for pore collapse in PMMA under a wide range of shock loading conditions. The underlying mechanics of pore collapse in different loading regimes is analyzed. In the past, the influence of loading strength on the pore collapse behavior in a condensed phase explosive (HMX) was analyzed using numerical simulations $[6,9,10,12,18]$. For high strength shocks, HMX was observed to melt under shock heating, leading to hydrodynamic collapse - characterized by material jetting [6,9]. The formation of a material jet is also observed in the collapse of pores in a liquid gel subjected to impulsive loading $[7,8,11$, 13]. For moderate to weak strength shocks, where the shock heating does not lead to the bulk melting of HMX, the collapse of the pore involves the formation of adiabatic shear bands [6]. Therefore, for solid materials that exhibit rate dependent plastic behavior, previous simulations have revealed a transition in the pore collapse mode with increasing shock strength.

To understand and establish the physics governing the transition behavior of the pores in solids, results from experiments and simulations for the collapse of a $6 \mathrm{~mm}$ diameter circular pore in PMMA are compared for four different shock strengths with corresponding flyer velocities: $V_{\text {flyer }}=0.40,0.59,1.70$ and $4.70 \mathrm{~km} / \mathrm{s}$. Table II lists the details of the experimental configurations. Using the properties of the flyer materials and PMMA target, the particle velocity generated upon impact is determined through impedance matching:

$$
U_{p, P M M A}=\frac{\left(\rho c_{o}\right)_{\text {flyer }}}{\left(\rho c_{o}\right)_{\text {flyer }}+\left(\rho c_{o}\right)_{P M M A}} V_{\text {flyer }}
$$

where, $\rho$ is the density and $c_{0}$ is the sound speed in the material. Using Eq. (18), the corresponding particle velocities in PMMA for the four loading strengths are calculated as $U_{p}=0.32,0.47,0.84$ and $2.30 \mathrm{~km} / \mathrm{s}$. Figure 3 shows the computational setup used to perform the simulations. The shock load is applied from the west face of the domain boundary using a rectangular pulse of duration, $t_{p}$,

$$
t_{p}=\frac{2 W_{\text {flyer }}}{U_{s}}
$$

where $W_{\text {flyer }}$ is the flyer thickness and $U_{s}$ is the shock speed in PMMA. The flyer thickness for the different loading configurations is given in Table II. Zero-gradient boundary conditions are applied for the east, north and south boundaries. For 3D simulations, the simulation set-up is analogous to 2D analysis with top and bottom boundaries supplied with zero-gradient boundary conditions.

It is important to note that experiments are performed for a spherical pore. Since 3D computations are expensive, the behavior of the spherical pore is simulated using the $2 \mathrm{D}$ circular pores of the same diameter. To assess the validity of the $2 \mathrm{D}$ approximation in the different loading regimes, two 3D simulations for the low, i.e. $U_{p}=0.32 \mathrm{~km} / \mathrm{s}$ and high, i.e. $2.30 \mathrm{~km} / \mathrm{s}$ strength shocks are performed. The 2D and 3D simulations are performed using a refined grid, of size $12 \mu \mathrm{m}$, which corresponds to 500 grid points across the pore diameter [9]. 
The results from the analysis are presented as follows: sections 3.1-3.3 present the comparison between the experimental and simulations results for the pore collapse under 4 shock strengths, i.e. $U_{p}=$ $0.32,0.47,0.84$ and $2.30 \mathrm{~km} / \mathrm{s}$. The underlying physics governing the collapse behavior of the pore is analyzed using the simulation results. Following the comparison, section 3.4 presents quantitative estimates of the pore collapse behavior by establishing scaling relationships between jet velocity and time to collapse as a function of loading strength. The scaling relations from the experiments and simulations are compared. The key findings and directions for future work resulting from the current analysis are presented in section 4.

\subsection{Pore collapse under shock strength at particle velocity of $0.32 \mathrm{~km} / \mathrm{s}$}

First, we study the pore collapse behavior of the $6 \mathrm{~mm}$ diameter pore in PMMA for a low shock strength corresponding to a particle velocity of $0.32 \mathrm{~km} / \mathrm{s}$. To assess the consequences of the 2D assumption, 3D pore collapse is also simulated; these simulation results are compared against corresponding experimental data.

Figure 4(a) compares the time variation of the normalized pore area obtained from experiment, and 2D and 3D simulations. For the experiment and the 3D simulation, the area of the pore is obtained for the central plane through the pore perpendicular to the shock direction as explained in section 2.4. The simulated response from the $2 \mathrm{D}$ and $3 \mathrm{D}$ calculations are both in close agreement with the experimental result. It is important to note that in Figure 4(a), the normalized pore area is shown up to a value of 0.5, i.e. corresponding to the halfway point of the pore closure. This is because, for the given loading condition, at this point of time in the experiment, the glue layer between the pore surfaces begins to separate. Therefore, complete collapse of the pore is not observed.

To gain insights into the pore collapse behavior, the transient pore profiles are extracted for direct comparison between the experiment and simulations data. The simulated pore profiles are shown overlaid on the experiment images in Figures 4(b) and 4(c) at the different time instants numbered from (i)-(v) in Figure 4(a). The simulations are in close agreement with experimental results during the collapse of the pore, with the 3D modeling showing subtle features of weak shock loading. The above comparison for the $U_{p}=0.32 \mathrm{~km} / \mathrm{s}$ case points out two general observations, a) the deformed pore profiles in Figures 4(b) and 4(c) show the formation of irregular ridges on the pore surface at later stages of the collapse, as seen in Figures 4(b,iv) and 4(b,v). These ridges are observed in both simulations and experiment, b) The pore profiles at the various instants shown are predicted well by both the 2D and 3D simulations. To understand the physics governing the ridge formation and to assess the validity of the $2 \mathrm{D}$ modeling assumption in detail, the simulation results are further analyzed.

The physical mechanism governing the ridge formation is analyzed using the temperature contours at different instants from the 2D simulation as shown in Figure 5. Figure 5(a) shows that the shock wave interaction with the pore leads to the initiation of adiabatic shear bands at the upper and lower pore surfaces. These shear bands have been simulated by other researchers in both energetic crystals $[6,10]$ as well as metals [39-41]. In the present calculations, the shear bands in PMMA are captured using the rate dependent Johnson Cook plasticity model [25]. The ridges on the pore surface are formed by the shear band initiation sites, as also seen in previous studies $[6,10,40]$. The shear band initiates and grows from the pore surface at an initial angle of $\approx 90^{\circ}$ to the shock direction as can be seen from Figure 5(b). At later times, the bands turn along the direction of the shock and the tips of the bands grow at an angle of $\approx 45^{\circ}$. This shows that the growth of bands is favored along the expected maximum shear stress plane, i.e. $45^{\circ}$. The localization of the plastic dissipation in the bands leads to temperatures in excess of the $400 \mathrm{~K}$, the melting point of PMMA [25]. The PMMA material melts in the band, leading to liquid PMMA bands sandwiched between the solid PMMA material, as can be seen from Figure 5(b). The initiation and growth of the bands is accompanied by the shrinking and compression of the pore from all directions under the incident shock 
load. Figure 5(c) shows that the compression of the pore eventually leads to its collapse followed by the generation of a diverging spherical re-shock wave. The re-shock wave propagates into the PMMA material and leads to further heating of the melted PMMA in the band causing temperature elevation of approximately $100 \mathrm{~K}$ as shown in Figure 5(d). The collapse of the pore for this $U_{p}=0.32 \mathrm{~km} / \mathrm{s}$ case forms a heated region consisting of a network of adiabatic shear bands around a central heated core corresponding to the collapse site. This structure of the hotspot has implications for initiation in reactive energetic materials where similar combination of collapse induced re-shock wave formation and heating of the shear bands can be expected.

From the above $2 \mathrm{D}$ analysis, the ridges on the pore surface in experiments are identified as the initiation sites for the adiabatic shear bands. Similar to the 2D and experiment results, the 3D pore profiles in Figure 4(b) also show the formation of ridges on the pore surface. To visualize the shear bands in 3D, Figure 6 shows the iso-surface of the effective plastic strain colored with the temperature contours. Along with the iso-surface of the effective plastic strain, the temperature on the centerline Z-axis plane and the deformed pore surface are also shown. Figure 6(a) shows that the interaction of the shock wave with the pore surface leads to the initiation of adiabatic shear bands at sites coinciding with the ridges observed in Figure 4(b). The bands initiate at an angle of $90^{\circ}$ and later grow along the maximum shear localization plane at approximately $45^{\circ}$ with respect to the incident shock direction as can be seen from Figures 6(b) and 6(c). This observation is consistent with the 2D results, except that in 3D, multiple bands grow outward as concentric petal-like formations. Figure 6(c) shows the surfaces of the petals. The increase in the temperature along the shear band surfaces melts the PMMA material. The pore shrinks and eventually collapses leading to the formation of a re-shock wave seen in Figure 6(d). The spherical re-shock wave propagates radially leading to increase in the temperature of the band surface by approximately $100 \mathrm{~K}$ as shown in Figures 6(e-g). The initiation process and profile of the adiabatic shear bands in the 2D and 3D analysis are qualitatively similar. However, the mass of the heated region from the plastic dissipation and the pore collapse is significantly higher in $3 \mathrm{D}$.

The present analysis shows that the numerical simulations in both $2 \mathrm{D}$ and $3 \mathrm{D}$ can predict the experimentally observed pore collapse behavior. To evaluate the influence of material strength effects on the pore collapse behavior, the collapse of a $6 \mathrm{~mm}$ dimeter pore under an increased shock strength corresponding to particle velocity, $U_{p}=0.47 \mathrm{~km} / \mathrm{s}$ is analyzed in the next section.

\subsection{Pore collapse for $U_{p}=0.47 \mathrm{~km} / \mathrm{s}$}

Figure 7(a) shows the comparison between the time variation of the normalized pore area between 2D simulation and experiments for $U_{p}=0.47 \mathrm{~km} / \mathrm{s}$; good agreement between the simulation and experimental results can be seen. It is important to note that the issue of the glue layer separation in the experimental results is not present in this higher shock strength case and the pore deformation data is available for later stages of collapse in the experiment. Figure 7(b) shows the overlaid plots of the pore profiles at different time instants numbered from (i) - (v) in Figure 7(a). The pore profiles predicted from the 2D simulation are in close agreement with the experimental results across different stages of collapse. It is interesting to note that similar to the previous case of $0.32 \mathrm{~km} / \mathrm{s}$ shock, Figure 7 (b) shows the formation of irregular ridges on the pore surface. The ridge formation in both simulation and experiment is observed at a later stage of pore collapse, i.e. $t>2.6 \mu \mathrm{s}$. The locations of the ridges also appear to match closely between the experiment and simulation results.

For this higher shock strength, the features of the pore collapse is observed for the previous lower velocity case seem to largely hold true as shown by the temperature contours in Figure 8 . The pore deformation progresses along with the surface ridges leading to the complete pore closure. In this case, however, the pore closure generates a stronger re-shock wave that propagates radially outward from the 
collapse site as shown in Figure 8(d). The re-shock wave leads to increase in temperature of the already melted bands by $\approx 150 \mathrm{~K}$.

The mechanism of pore collapse for the $0.47 \mathrm{~km} / \mathrm{s}$ shock is similar to the $0.32 \mathrm{~km} / \mathrm{s}$ case. For both these shock strengths, the collapse is dominated by strength effects and plastic dissipation. The resulting hotspot region consists of a network of shear bands surrounding a heated core region corresponding to the location of the final pore closure site. Although there are similarities in the pore collapse mechanism for the two shock strengths, the locations of the band initiation sites and the morphology of the shear band network are significantly different between the two cases. First, for the $0.32 \mathrm{~km} / \mathrm{s}$ shock, the adiabatic shear bands are initiated near the equator on the upper and lower surfaces of the pore and they initially start to grow at an angle of $90^{\circ}$ to the pore surface as can be seen from Figure 5(b). On the other hand, for the 0.47 $\mathrm{km} / \mathrm{s}$ case, the adiabatic shear bands are seeded at an angle of $135^{\circ}$ with the shock propagation direction. In addition, the band morphology for the two cases are also different. For the $0.47 \mathrm{~km} / \mathrm{s}$ shock, the gaps between the adjacent bands at the pore surface are observed to be less than the $0.32 \mathrm{~km} / \mathrm{s} \mathrm{case}$. This leads to the formation of a finer band network as can be seen by comparing Figures 8(b) and 5(b). Since the shear band density is higher for the $0.47 \mathrm{~km} / \mathrm{s}$, the hotspot mass is also higher for the higher shock strength. In the case of energetic materials, this increase of hotspot mass can lead to faster hotspot reaction initiation and growth rates.

\subsection{Pore collapse under higher shock strengths, $U_{p}=0.84 \mathrm{~km} / \mathrm{s}$ and $2.30 \mathrm{~km} / \mathrm{s}$}

We now examine the dynamics of pore collapse for higher shock strengths, in the regime where inertia is expected to play the dominant role. For a shock corresponding to $U_{p}=0.84 \mathrm{~km} / \mathrm{s}$, Figure 9(a) compares the time variation of the normalized pore area obtained from the $2 \mathrm{D}$ simulation and experiment. As seen in the figure, there is good agreement between the experiment and simulation observed during the initial stages of pore collapse, i.e. $t<1.5 \mu$ s. The calculated pore area deviates from the experimental results for $t>1.5 \mu \mathrm{s}$. To understand this deviation, the deformed pore profiles from simulation and experiment are overlaid in Figure 9(b) at different instants numbered (i) through (v). Figures 9(b,i)-9(b,iii) show that for $t<1.5 \mu \mathrm{s}$, the pore profiles from the simulation closely match the experimental results. At later times, as shown in Figures 9(b,iv)-9(b,v), the simulated deformation of the pore front surface along the centerline is higher; in the experiments, the front surface of the pore is observed to be flatter, i.e. the jet formation is less prominent in the experiment. This difference in shape of the pore in the later stages of collapse leads to the lower value of computed normalized pore area in Figure 9(a).

For the $U_{p}=0.84 \mathrm{~km} / \mathrm{s}$ case, bulk shock heating in PMMA leads to a temperature rise of $\approx 450 \mathrm{~K}$, and PMMA melts under the incident shock load as can be seen from Figure 10(a). Unlike the previous 0.32 $\mathrm{km} / \mathrm{s}$ and $0.47 \mathrm{~km} / \mathrm{s}$ cases, no ridge formation on the pore surface is observed. The pore collapse progresses in liquid PMMA and the interaction of the incident shock with pore surface leads to the formation of a material jet as shown in Figure 10(b). The shock focusing by the material jet causes larger deformation along the centerline of the pore. Jet impact at the centerline leads to the generation of a re-shock wave and two symmetric lobes as can be seen from Figure 10(b). The lobes collapse under the combined influence of the re-shock wave and the incident shock. Figure 10(c) shows that the complete closure of the pore leads to the formation of a mushroom shaped intense hotspot. The peak temperature in the hotspot region is approximately $1000 \mathrm{~K}$ higher than the $0.47 \mathrm{~km} / \mathrm{s}$ case; this can be seen by comparing Figures $10(\mathrm{~d})$ and $8(d)$.

The $0.84 \mathrm{~km} / \mathrm{s}$ case above is seen to lead to a hydrodynamic jetting mode of collapse of the pore. To examine collapse at even higher velocities, we compare simulation and experimental results for the highest flyer velocity tested, corresponding to $U_{p}=2.30 \mathrm{~km} / \mathrm{s}$. In this case, as for the lowest velocity case of $U_{p}=0.32 \mathrm{~km} / \mathrm{s}$, both 2D and 3D calculations are performed to assess the impact of performing $2 \mathrm{D}$ as opposed to 3D calculations. 
Figure 11(a) shows the comparison of the time variation of the normalized pore area between the experimental results and the 2D and 3D simulation results. The normalized pore area calculated from the 2D simulation is in close agreement with the experimental data. This contrasts with the deviations observed in the $0.84 \mathrm{~km} / \mathrm{s}$ case. To further validate the pore collapse behavior, we overlay deformed pore profile plots at several time instants (i) to (v) in Figure 11(b); the corresponding instants on the pore area versus time plot can be seen in Figure 11(a). Figures 11(b,i)-11(b,iii) show that for $t<0.75 \mu s$, the deformed pore profile obtained from the simulation is nearly identical to the experimental results. At later stages, i.e. for $t>0.75 \mu \mathrm{s}$, the centerline deformation of the front pore surface is lower than the experimental value. To understand the influence of the dimensionality on the deformed profile, a high-fidelity 3D pore collapse simulation for a $6 \mathrm{~mm}$ diameter spherical pore is performed. Figure 11(a) shows the comparison of the normalized pore area obtained from the 3D calculation with the experimental data. The normalized pore area predicted from the 3D simulation matches well with the experiments. Similar to the 2D overlaid plots, Figure 11(c) shows the comparison between the deformed pore profiles obtained from the 3D simulation with experimental results at the instants (i) through (v). The 3D pore profiles are almost identical in the early as well as later stages of the collapse as can be seen from Figures 11(c). It is noteworthy that at later stages, unlike in the 2D simulations, the front surface of the pore shows nearly the same deformation in 3D as experiments as shown in Figures 11(c,iv) - 11(c,v). Therefore, 3D simulations produce better agreement with the experiments in terms of the details of the pore shape over the entire collapse process, when compared to the 2D simulations. However, gross measures, such as pore area and collapse time obtained from 2D calculations are in good agreement with experiments.

For the $U_{p}=2.30 \mathrm{~km} / \mathrm{s}$ case, Figures 12 shows the temperature contours at different instants in the 2D and 3D simulations. The shock heating in the bulk of PMMA leads to the increase in temperature to $\sim 1200 \mathrm{~K}$. The bulk PMMA material therefore melts for the $2.30 \mathrm{~km} / \mathrm{s}$ loading condition; similar to the $0.84 \mathrm{~km} / \mathrm{s}$ case, the collapse of the pore progresses in a liquid PMMA material. The shock interaction with the pore leads to the formation of a material jet as can be seen from Figure 12(a,i). The jet impact at the centerline forms two sidelobes and generates a re-shock wave that propagates radially outward from the impact location as shown in Figure 12(a,ii). Under the combined influence of the re-shock wave and incident shock, the lobes collapse leaving behind a mushroom-shaped heated region as can be seen from Figure 12(a,iii). Because of the misalignment in the pressure and density gradients during the collapse of the lobes, barotropic vortices are generated. This draws the hotspot into a slender, convoluted shape. It is interesting to note that the hotspot shape is similar to the $0.84 \mathrm{~km} / \mathrm{s}$ case with elevated temperature. Comparing the final hotspot shapes for the four different velocities used in this study, i.e. in Figures 5(d), 8(d), 10(d) and 12(a,iii), it can be seen that the hotspots have highly irregular, convoluted shapes and are surrounded by substantial areas of material at elevated temperatures. These characteristics of the hotspots have implications for the initiation and growth of hotspots in energetic materials.

In the 3D pore collapse process, the mechanism of jet formation at the centerline pore surface is similar to the 2D case. The key difference lies in the collapse of the lobes where the barotropic vortices are formed in the 3 dimensions. To visualize these barotropic vortices in $3 \mathrm{D}$, the iso-surface of the vorticity (i.e. isocontours of the second invariant of the rotation tensor, $\lambda_{2}$ ) colored with the temperature contours are shown at different instants in Figure 12(b). Along with the vortices contours, Figure 12(b) shows the temperature contours along the centerline Z-plane and the deformed pore surface. Figure 12(b,i) shows that the incident shock deforms the spherical pore and leads to the formation of a material jet along the centerline of the pore. The shock focusing because of material jetting leads to the pore closure at the centerline and generates a re-shock wave from the jet impact site. The combined effect of the re-shock wave and the incident shock eventually leads to the complete closure of the pore with generation of the barotropic vortex rings as shown in Figure 12(b,iii). The core of the vortex rings coincides with the maximum temperature 
rise from the collapse. The rings translate along the shock direction and eventually disintegrate and dissipate leaving behind a mushroom shaped heated region in 3D.

The comparison between the $2 \mathrm{D}$ and $3 \mathrm{D}$ results shows that $2 \mathrm{D}$ simulations can qualitatively calculate pore collapse in the hydrodynamic regime. However, the hotspot mass in 3D is higher which can lead to enhanced ignition and growth rates in 3D when compared to 2D. Furthermore, the higher centerline pore deformation in 3D and experiments seen in Figures 11(b) and 11(c) indicates that the jet speed in 2D is underpredicted.

\subsection{Relationship between pore collapse measures and incident shock strength}

In the previous sections, the collapse behavior of the pores in various loading regimes is analyzed and explained using experiments and numerical simulations. In the present section, using the experimental and simulation data for various shock strengths, quantitative measures describing the collapse process and its relation to the loading strength is established.

As explained in section 2.4, from the experiments and simulation results, various quantitative measures including normalized pore area (Eq. (10)) and displacement, $x$ of the pore interface along the pore centerline (Eq. (11)) can be used to estimate time to collapse, velocity and acceleration of the pore interface. Using the method described earlier, these measures are obtained from the experiments and simulations data and scaling relations describing the collapse behavior across wide range of loading regimes are established. In the past [11], such relations are reported for liquids and gels where the collapse mode is purely hydrodynamic. To compare the current results with the previously reported relations, and to obtain a reliable fit from the simulation results, four additional 2D pore collapse simulations are performed (in the hydrodynamic regime) under shock loads corresponding to $U_{p}=1.10,1.4,1.70$ and $2 \mathrm{~km} / \mathrm{s}$.

Figure 13(a) shows the variation of the dimensionless time to collapse with the incident shock pressure ratio, calculated from the simulations and experiments. The pressure ratio is dimensionless, and given by $p^{\prime}=p / p_{0}$, where $p_{0}=1$ atm is the ambient atmospheric pressure, and the dimensionless time is as defined earlier in Eq. (12). The black line in the plot, which is log-log, shows the fit of the data analyzed by Escauriza et al. [22]. It was found that above $1.85 \mathrm{GPa}$ there is a power law relationship between $t^{\prime}$ and $p^{\prime}$ that, interestingly, spans the pressure range from the strength regime to the maximum pressure studied in the hydrodynamic regime of $16.60 \mathrm{GPa}$. The relationship is given by the expression,

$$
t_{A, \text { min,experiments }}^{\prime}=498 p^{-0.58}
$$

which is very similar to a relationship to that discovered by Swantek and Austin [11] in their study on dynamically loaded cylindrical pores in hydrogel. The expression is also close to the analytic inverse root relationship between the collapse time and ambient fluid pressure for bubble collapse, derived by Rayleigh [42]. This collapse time, which is coupled with the jet dynamics described earlier, is related to the temperature and pressure states produces during collapse.

The collapse time power law curve calculated from the $2 \mathrm{D}$ simulations, which is shown in the plot as a dashed red line, is in close agreement with the experimental estimation for lower pressures. For higher pressures, the 2D simulations diverge and estimate higher collapse time as compared to the experiments. This was observed in section 3.3 for the $2.30 \mathrm{~km} / \mathrm{s}$ shock, where the less deformation along the pore centerline in the $2 \mathrm{D}$ simulation was observed compared to the experiments. The collapse time estimation from 3D simulation and experiments for $2.30 \mathrm{~km} / \mathrm{s}$ shock were observed to be in close agreement, and this is reflected in Figure 13(a). The fits from the experiments and simulations are obtained as,

$$
t_{A, \min , 2 \mathrm{Dsim}}^{\prime}=191 p^{-0.49}
$$


It is interesting to note Swantek and Austin [11] obtained a similar relationship by performing experiments on a gel material under shock, with the collapse time scaling with the pressure ratio by a -0.55 exponent, i.e. close agreement with the current estimations for PMMA.

An additional metric to quantify the dynamics of the pore collapse behavior as a function of the loading strength is the jet velocity and acceleration for the pore interface along the centerline, which are calculated using Eqs. (12-17). As discussed in Section 2.4, the acceleration was only calculated for pressures $\geq 4.80 \mathrm{GPa}$. Figure 13(b) shows that the acceleration of the pore centerline varies linearly with the loading pressure for both experiments and simulations. The linear fits for normalized pore interface acceleration for experiments and 2D simulations are obtained as,

$$
\begin{gathered}
a_{\text {experiments }}^{\prime}=0.38 p-0.93 \\
a_{2 \text { Dim }}^{\prime}=0.14 p+0.51
\end{gathered}
$$

Similar to the calculation of the centerline pore interface acceleration, using Eq. (17), the jet velocity at the time of the pore closure near the centerline is calculated for various shock strengths. Figure 13(c) shows the linear variation of the jet velocity with respect to the particle velocity for both simulations and experiments. The linear fit for the jet velocity as a function of shock particle velocity is obtained, from Eq. (17), as,

$$
\begin{gathered}
V_{\text {jet,experments }}=3.99 U_{p}-0.59 \\
V_{\text {jet,2Dsimulations }}=2.21 U_{p}+1.58
\end{gathered}
$$

where $V_{j e t}$ is the jet velocity and $U_{p}$ is the particle velocity.

Figure 13 (c) also shows the initial release velocities of the interface from the experiments and simulations, as a function of particle velocity. There is good agreement for both the $2 \mathrm{D}$ simulations and the single 3D simulation data point in the range studied. The two equations for the fitted line are,

$$
\begin{gathered}
V_{\text {release,experments }}=1.49 U_{p}+0.40 \\
V_{\text {release, } 2 \text { Dsimulations }}=1.75 U_{p}-0.15
\end{gathered}
$$

where $V_{\text {rel }}$ is the release velocity.

Figures 13(b) and (c) show that while the initial velocity of the interface is approximately equal, the jet velocity and acceleration of the centerline pore interface is higher in experiments as compared to the 2D simulations. However, in the 3D simulation, for $2.30 \mathrm{~km} / \mathrm{s}$ case, the results are in close agreement with the experiments. Therefore, the lower jet velocity and acceleration of the pore interface in the 2D simulation is attributed because of the pore geometry in 2D, i.e. cylindrical as opposed to spherical. Therefore, spherical pore leads to higher jet focusing and eventually manifests in higher collapse temperatures and intense hotspots. 


\section{Conclusions}

Head-to-head comparison between the experiments and simulations are performed to analyze the shock induced pore collapse behavior in PMMA for four shock strengths corresponding to particle velocities in the PMMA of $0.32,0.47,0.84$ and $2.30 \mathrm{~km} / \mathrm{s}$. The numerical simulations are performed using a Eulerian levelset based sharp-interface code SCIMITAR3D. The experiments are performed at the European Synchrotron Research Facility using single and two-stage gas guns, where ultra-high-speed Xray imaging was used to obtain experimental data and visualization of the pore during the collapse process. The numerical simulations approximate the experimental spherical pore geometry using a 2D circular pore of the same diameter as in experiments, i.e. $6 \mathrm{~mm}$. Since 3D simulations are expensive, high resolution spherical pore collapse simulations are performed for only 2 shock strengths of 0.32 and $2.30 \mathrm{~km} / \mathrm{s}$.

For all four shock strengths, the predicted pore profiles from simulations are in close agreement with experiments. The numerical framework can capture the mechanics of pore collapse for the different loading regimes with good physical fidelity. For $0.32 \mathrm{~km} / \mathrm{s}$ and $0.47 \mathrm{~km} / \mathrm{s}$ shock strengths, the experiments show asymmetric collapse with the formation of ridges on the pore surface. Using the numerical simulations, the ridges are identified as the locations of the initiation sites of the adiabatic shear bands that emanate from the pore surface. The collapse under low shock strengths is dominated by the rate dependent plastic dissipation. For higher shock strengths, i.e. 0.84 and $2.30 \mathrm{~km} / \mathrm{s}$, both experimental and simulation results show a symmetric pore collapse with no ridge formation. Under high speed loading, the PMMA melts because of shock heating and the pore effectively collapses in a liquid PMMA material, in a purely hydrodynamic mode with jet formation and generation of barotropic vortices. Between the two loading extremes, the collapse transitions from a strength dominated regime to a hydrodynamic regime. This transition is observed to set in the current analysis for loading pressure greater than $4.8 \mathrm{GPa}$, i.e. particle speed $0.8 \mathrm{~km} / \mathrm{s}$. The prediction of the transition regime in the numerical simulation is dependent on the a) accuracy of melting temperature, which is a function of pressure and b) temperature and pressure dependent specific heat value; more accurate models for melting temperature and specific heat will be required to pinpoint the transition load. The transition point may also depend on pore size. These issues remain matters of future investigation.

In the two different loading regimes, i.e. strength-dominated and hydrodynamic, the key differences between the 2D and 3D simulations are analyzed. The 2D simulations are observed to capture the qualitative behavior of the pore collapse in both loading regimes. For the lowest shock strength studied, the $0.32 \mathrm{~km} / \mathrm{s}$ case, in 3D, the adiabatic shear bands are formed in the form of concentric disks around the pore surface. The bands grow leading to a complex hotspot structure with a central heated core corresponding to the collapse site. The collapse site is surrounded by what appear to be layers of adiabatic shear band surfaces. On the other hand, for the $2.30 \mathrm{~km} / \mathrm{s}$ shock, the centerline pore deformation is observed to be higher in 3D and in experiments than 2D. Overall, the 3D simulations are in closer agreement with experiments; however, 2D simulations are far less computationally expensive which allows for exploration of parameter spaces for pore collapse and for construction of surrogate models, as done in previous work [14, 43, 44].

Finally, for quantitative comparison between the simulation and experiments data, scaling relations governing the pore collapse and shock strength are obtained and compared. The empirical relations for the time to collapse and pore surface acceleration as a function of input pressure are obtained. The quantitative relationships from the simulations are in close agreement with experiments. Furthermore, a useful scaling relationship between the hydrodynamic collapse jet speed and the imposed particle velocity is computed.

The present study establishes confidence in the numerical simulations to study the physics governing the pore collapse behavior in condensed phase solids over a wide range of loading conditions. Further ongoing work is directed towards clarifying the detailed physics of shear band emergence at the pore surface and the late-stage coarsening, bending and curvature of the shear bands. We are also investigating the way in which the energy content and temperature distribution in the hotspot depends on 
the occurrence or non-occurrence (in the hydrodynamic regime) of shear bands; the implications of these hotspot characteristics are expected to be significant for their initiation and growth and therefore for the sensitivity of energetic materials.

\section{Acknowledgements}

The authors gratefully acknowledge the financial support from the Air Force Research Laboratory Munitions Directorate (AFRL/RWML), Eglin AFB, under contract number FA8651-16-1-0005 (Program Manager: Dr. Angela Diggs). The contribution of Emilio M. Escauriza was funded by a joint European Synchrotron Radiation Facility (ESRF) and First Light Fusion studentship.

\section{References}

[1] J. Field, The physics of liquid impact, shock wave interactions with cavities, and the implications to shock wave lithotripsy, Physics in Medicine \& Biology 36 (1991) 1475.

[2] T. Kodama, K. Takayama, Dynamic behavior of bubbles during extracorporeal shock-wave lithotripsy, Ultrasound in medicine \& biology 24 (1998) 723-738.

[3] G. Sankin, P. Zhong, Interaction between shock wave and single inertial bubbles near an elastic boundary, Physical Review E 74 (2006) 046304.

[4] R.J. Browning, E. Stride, Microbubble-mediated delivery for cancer therapy, J Fluids 3 (2018) 74.

[5] J.E. Field, Hot spot ignition mechanisms for explosives, Accounts of Chemical Research 25 (1992) 489-496.

[6] H.K. Springer, S. Bastea, A.L. Nichols III, C.M. Tarver, J.E. Reaugh, Modeling the effects of shock pressure and pore morphology on hot spot mechanisms in HMX, Propellants, Explosives, Pyrotechnics 43 (2018) 805-817.

[7] L. Michael, N. Nikiforakis, The evolution of the temperature field during cavity collapse in liquid nitromethane. Part I: inert case, Shock Waves 29 (2019) 153-172.

[8] L. Michael, N. Nikiforakis, The evolution of the temperature field during cavity collapse in liquid nitromethane. Part II: reactive case, Shock Waves 29 (2019) 173-191.

[9] N.K. Rai, M.J. Schmidt, H.S. Udaykumar, High-resolution simulations of cylindrical void collapse in energetic materials: Effect of primary and secondary collapse on initiation thresholds, Physical Review Fluids 2 (2017).

[10] R.A. Austin, N.R. Barton, J.E. Reaugh, L.E. Fried, Direct numerical simulation of shear localization and decomposition reactions in shock-loaded HMX crystal, Journal of Applied Physics 117 (2015) 185902.

[11] A. Swantek, J. Austin, Collapse of void arrays under stress wave loading, Journal of Fluid Mechanics 649 (2010) 399-427.

[12] A. Kapahi, H.S. Udaykumar, Dynamics of void collapse in shocked energetic materials: physics of void-void interactions, Shock Waves 23 (2013) 537-558.

[13] N. Bourne, J. Field, Shock-induced collapse of single cavities in liquids, Journal of Fluid Mechanics 244 (1992) 225-240.

[14] S. Roy, N. Rai, O. Sen, D. Hardin, A. Diggs, H. Udaykumar, Modeling mesoscale energy localization in shocked HMX, Part II: training machine-learned surrogate models for void shape and void-void interaction effects, Shock Waves, (2019) 1-23.

[15] X. Mi, L. Michael, E. Ioannou, N. Nikiforakis, A.J. Higgins, H.D. Ng, Meso-resolved simulations of shock-to-detonation transition in nitromethane with air-filled cavities, Journal of Applied Physics 125 (2019) 245901.

[16] M. Armstrong, E. Bukovsky, W. Shaw, L. Lauderbach, R. Austin, P. Grivickas, R. Hodgin, N. Sinclair, J. Klug, T. Willey, Time-resolved X-ray Imaging of Void Collapse in Silicone and TNT, Lawrence Livermore National Lab.(LLNL), Livermore, CA (United States), 2018. 
[17] C.A. Duarte, A. Hamed, J.D. Drake, C.J. Sorensen, S.F. Son, W.W. Chen, M. Koslowski, Void Collapse in Shocked-HMX Single Crystals: Simulations and Experiments, Propellants, Explosives, Pyrotechnics 45 (2020) 243-253.

[18] D.B. Hardin, J.O. Mares. Temperature rise from collapsing pores. AIP Conference Proceedings; 2018: AIP Publishing. p. 180003.

[19] S. Sambasivan, A. Kapahi, H.S. Udaykumar, Simulation of high speed impact, penetration and fragmentation problems on locally refined Cartesian grids, Journal of Computational Physics, (2012).

[20] A. Kapahi, S. Sambasivan, H. Udaykumar, A three-dimensional sharp interface Cartesian grid method for solving high speed multi-material impact, penetration and fragmentation problems, Journal of Computational Physics 241 (2013) 308-332.

[21] N.K. Rai, A. Kapahi, H.S. Udaykumar, Treatment of contact separation in Eulerian high-speed multimaterial dynamic simulations, International Journal for Numerical Methods in Engineering 100 (2014) 793-813.

[22] E. Escauriza, J. Duarte, D. Chapman, M. Rutherford, L. Farbaniec, J. Jonsson, L. Smith, M. Olbinado, J. Skidmore, P. Foster, Collapse dynamics of spherical cavities in a solid under shock loading, Scientific Reports 10 (2020) 1-16.

[23] A.S. Khan, S. Huang, Continuum Theory of Plasticity, John Wiley \& Sons1995.

[24] J. Simo, T. Hughes, General return mapping algorithms for rate-independent plasticity, Constitutive laws for engineering materials: theory and applications 1 (1987) 221-232.

[25] T. Holmquist, J. Bradley, A. Dwivedi, D. Casem, The response of polymethyl methacrylate (PMMA) subjected to large strains, high strain rates, high pressures, a range in temperatures, and variations in the intermediate principal stress, The European Physical Journal Special Topics 225 (2016) 343-354.

[26] G.S. Collins, D. Elbeshausen, K. Wünnemann, T.M. Davison, B. Ivanov, H.J. Melosh, iSALE: A multi-material, multi-rheology shock.

[27] S.P. Marsh, LASL shock Hugoniot data, Univ of California Press1980.

[28] M. Blackman, The theory of the specific heat of solids, Reports on Progress in Physics 8 (1941) 11.

[29] C.-W. Shu, S. Osher, Efficient Implementation of Essentially Non-Oscillatory Shock-Capturing Schemes, II, Journal of Computational Physics 83 (1989) 32-78.

[30] S. Osher, J.A. Sethian, Fronts Propagating with Curvature-Dependent Speed - Algorithms Based on Hamilton-Jacobi Formulations, Journal of Computational Physics 79 (1988) 12-49.

[31] R.P. Fedkiw, T. Aslam, B. Merriman, S. Osher, A non-oscillatory Eulerian approach to interfaces in multimaterial flows (the ghost fluid method), Journal of Computational Physics 152 (1999) 457-492.

[32] N.K. Rai, H.S. Udaykumar, Mesoscale simulation of reactive pressed energetic materials under shock loading, Journal of Applied Physics 118 (2015) 245905.

[33] M.E. Rutherford, D.J. Chapman, J.G. Derrick, J.R. Patten, P.A. Bland, A. Rack, G.S. Collins, D.E. Eakins, Probing the early stages of shock-induced chondritic meteorite formation at the mesoscale, Scientific reports 7 (2017) 45206.

[34] E.M. Escauriza, M.P. Olbinado, M.E. Rutherford, D.J. Chapman, J.C. Jonsson, A. Rack, D.E. Eakins, Ultra-high-speed indirect $\mathrm{x}$-ray imaging system with versatile spatiotemporal sampling capabilities, Applied Optics 57 (2018) 5004-5010.

[35] S. Wilkins, T.E. Gureyev, D. Gao, A. Pogany, A. Stevenson, Phase-contrast imaging using polychromatic hard X-rays, Nature 384 (1996) 335-338.

[36] P. Cloetens, R. Barrett, J. Baruchel, J.-P. Guigay, M. Schlenker, Phase objects in synchrotron radiation hard x-ray imaging, Journal of Physics D: Applied Physics 29 (1996) 133.

[37] J. Canny, A computational approach to edge detection, IEEE Transactions on pattern analysis machine intelligence, (1986) 679-698.

[38] Y.V. Bat'kov, S. Novikov, N. Fishman. Shear stresses in polymers under shock compression. AIP Conference Proceedings; 1996: American Institute of Physics. p. 577-580.

[39] V. Nesterenko, M. Meyers, T. Wright, Self-organization in the initiation of adiabatic shear bands, Acta materialia 46 (1998) 327-340. 
[40] Q. Xue, M. Meyers, V. Nesterenko, Self-organization of shear bands in titanium and Ti-6Al-4V alloy, Acta Materialia 50 (2002) 575-596.

[41] N. Kudryashov, P. Ryabov, A. Zakharchenko, Self-organization of adiabatic shear bands in OFHC copper and HY-100 steel, Journal of the Mechanics Physics of Solids 76 (2015) 180-192.

[42] L. Rayleigh, On the pressure developed in a liquid during the collapse of a spherical cavity:

Philosophical Magazine Series 6, 34, 94-98, 1917.

[43] A. Nassar, N.K. Rai, O. Sen, H. Udaykumar, Modeling mesoscale energy localization in shocked HMX, part I: machine-learned surrogate models for the effects of loading and void sizes, Shock Waves, (2018) 1-22.

[44] O. Sen, N. Rai, A. Diggs, D. Hardin, H. Udaykumar, Multi-scale shock-to-detonation simulation of pressed energetic material: A meso-informed ignition and growth model, Journal of Applied Physics 124 (2018) 085110. 


\begin{tabular}{|c|c|}
\hline \multicolumn{2}{|c|}{ PMMA Material Properties } \\
\hline Density, $\rho\left(\mathrm{kg} / \mathrm{m}^{3}\right)$ & 1186 \\
\hline Speed of Sound, $c_{o}(\mathrm{~m} / \mathrm{s})$ & 2230 \\
\hline Shear Modulus, $G(\mathrm{GPa})$ & 5.9 \\
\hline $\begin{array}{c}\text { Isochoric Specific Heat } \\
\text { Capacity, } C_{v}(\mathrm{~J} / \mathrm{Kg} \mathrm{k}) \text { at } 298 \mathrm{~K}\end{array}$ & 1466 \\
\hline Johnson-Cook $(\mathrm{JC})$ & Model Parameters \\
\hline$A(\mathrm{MPa})$ & 357 \\
\hline$B(\mathrm{MPa})$ & 90 \\
\hline$n$ & 4 \\
\hline$C$ & 0.077 \\
\hline$m$ & 0.74 \\
\hline Melting Temperature, $T_{M E L T}(\mathrm{~K})$ & 398 \\
\hline Tillotson Equation of State Parameters \\
\hline$a$ & 0.85 \\
\hline$b$ & 0.0 \\
\hline$E_{0}(\mathrm{~J} / \mathrm{Kg})$ & $7.95 e 6$ \\
\hline$A(\mathrm{GPa})$ & 5.9 \\
\hline$B(\mathrm{GPa})$ & 19 \\
\hline$\alpha$ & 0.0 \\
\hline$\beta$ & 0.0 \\
\hline
\end{tabular}

Table I: Constitutive properties of PMMA used in the pore collapse simulations $[25,27]$

\begin{tabular}{|c|c|c|c|c|c|c|}
\hline Flyer material & $\begin{array}{c}\text { Pore } \\
\text { Diameter } \\
(\mathrm{mm})\end{array}$ & $\begin{array}{c}\text { Flyer } \\
\text { Thickness } \\
(\mathrm{mm})\end{array}$ & $\begin{array}{c}\text { Flyer speed } \\
(\mathrm{km} / \mathrm{s})\end{array}$ & $\begin{array}{c}\text { Particle } \\
\text { velocity } \\
(\mathrm{km} / \mathrm{s})\end{array}$ & $\begin{array}{c}\text { Shock } \\
\text { velocity } \\
(\mathrm{km} / \mathrm{s})\end{array}$ & $\begin{array}{c}\text { Pressure } \\
(\mathrm{GPa})\end{array}$ \\
\hline Aluminum & 6 & 2 & $0.40 \pm 0.01$ & $0.32 \pm 0.01$ & $3.23 \pm 0.10$ & $1.21 \pm 0.04$ \\
\hline Aluminum & 6 & 2 & $0.59 \pm 0.01$ & $0.47 \pm 0.02$ & $3.31 \pm 0.10$ & $1.84 \pm 0.06$ \\
\hline Polycarbonate & 6 & 2 & $1.70 \pm 0.01$ & $0.84 \pm 0.03$ & $3.87 \pm 0.12$ & $4.10 \pm 0.12$ \\
\hline Polycarbonate & 6 & 2 & $4.70 \pm 0.02$ & $2.30 \pm 0.07$ & $6.09 \pm 0.19$ & $16.60 \pm 0.49$ \\
\hline
\end{tabular}

Table II: Pore collapse experiments details for $6 \mathrm{~mm}$ diameter pore under four different shock strengths 

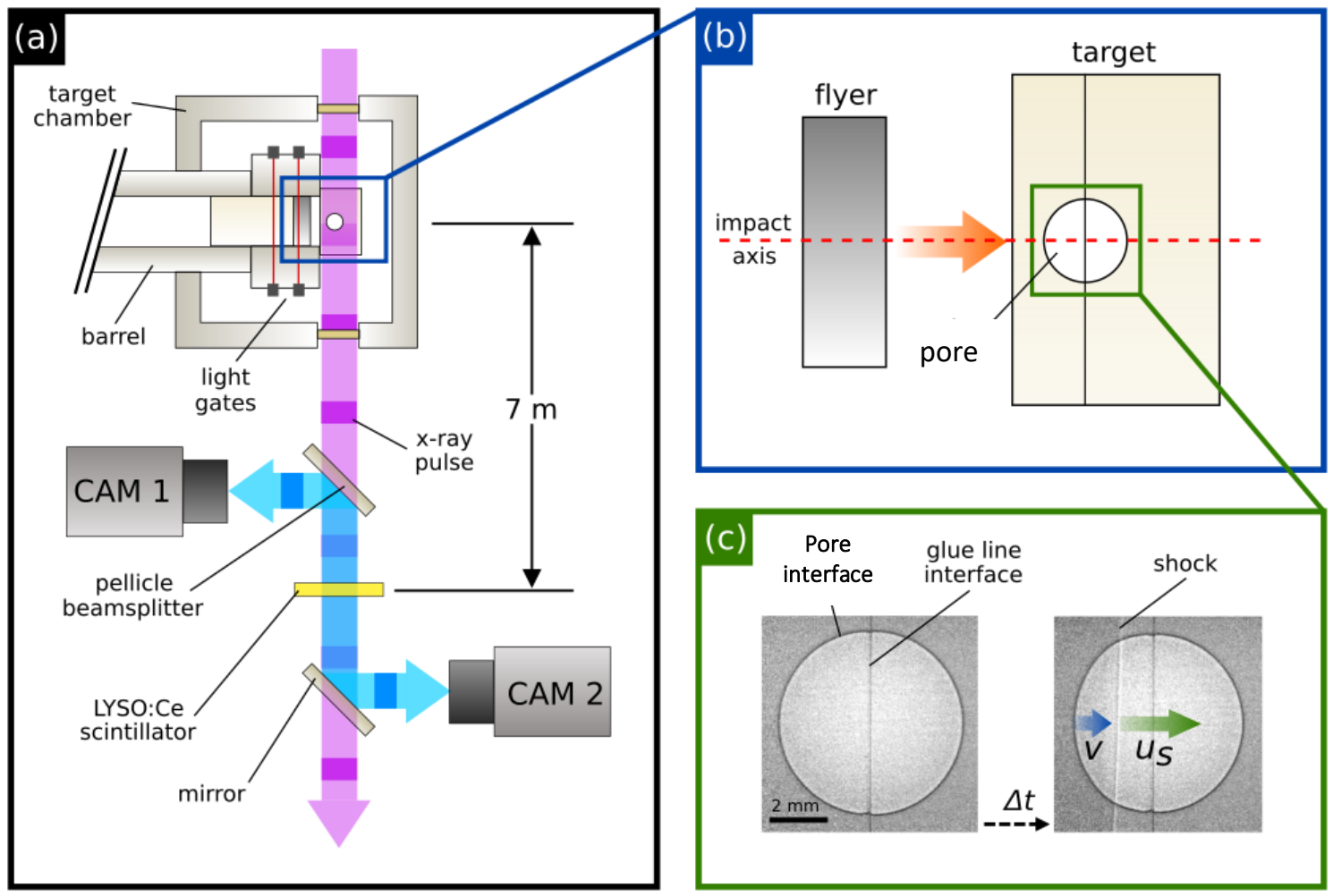

Figure 1: Schematic diagram showing details of the experimental setup. (a) The X-rays propagate through the target chamber normal to the impact direction. The imaging system is placed $7 \mathrm{~m}$ away from the target in the region of phase contrast enhancement. The X-rays are partially absorbed by the scintillator and re-emitted in visible light, which is captured with two high-speed cameras. (b) Section view of the impact between the flyer and pore target, which is symmetric about the impact axis. The target is constructed from two cylindrical sections glued

together. (c) Example radiographs showing a section view of the pore before and during interaction with the shock. The arrows indicate the pore interface velocity, $\mathrm{v}$, and the shock velocity, $\mathrm{U}_{\mathrm{s}}$. 


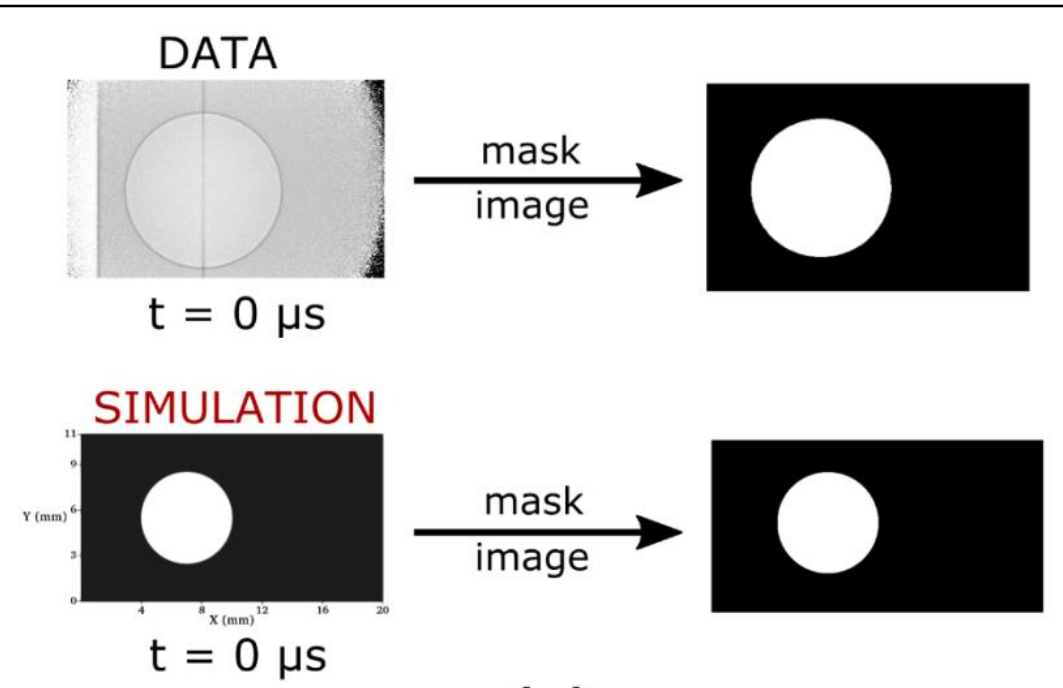

(a)
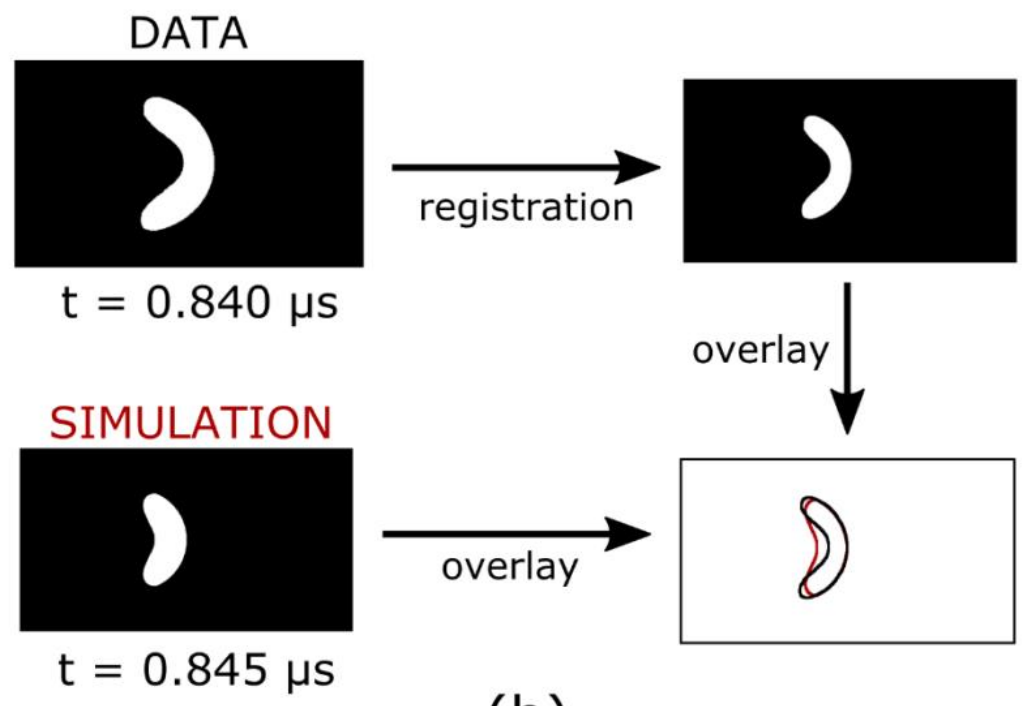

(b)

Figure 2: Schematic diagram showing the procedure to generate overlay plots between the experiment and simulation plots of pore deformation. a) A mask image of the pore is made from the $2 \mathrm{D}$ radiographic image and simulation image at initial time, $t=0 \mu \mathrm{s}$. These two images are used to obtain the registration transform parameters. (b) Each frame of the data mask images is transformed through image registration and an overlay image is created by matching to a simulation image mask at the same approximate time. 


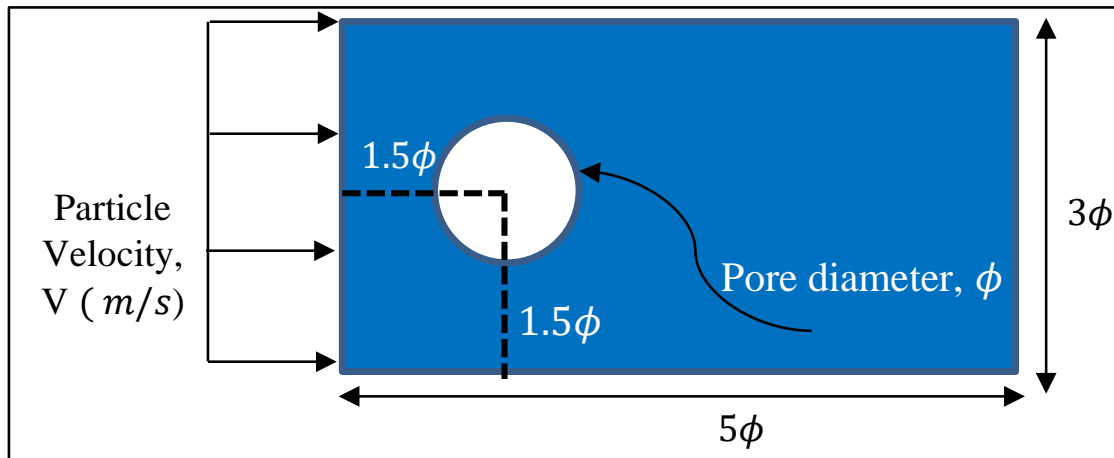

Figure 3: Numerical set up for pore collapse simulation of cylindrical pores. Shock load is applied as a velocity pulse from the west domain boundary. Zero gradient boundary conditions are applied for the east, north and south domain boundary. 


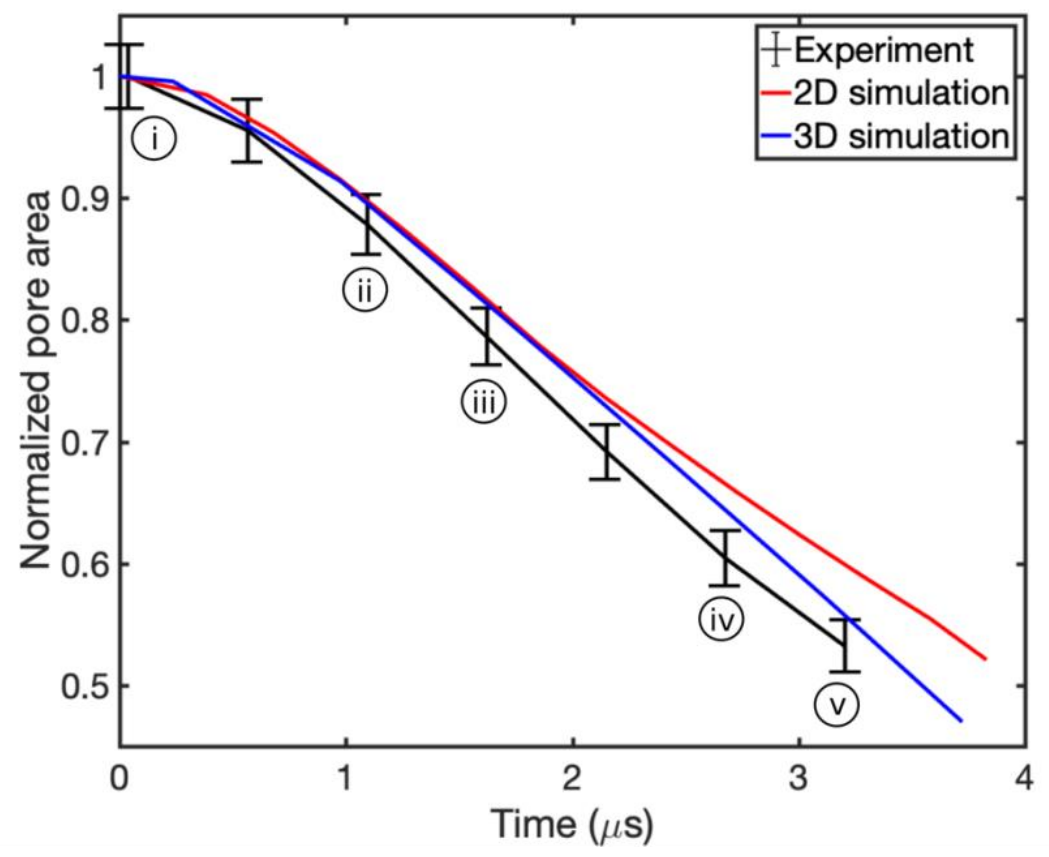

(a) Experimental and simulation results comparison of the time evolution of the normalized pore area for $6 \mathrm{~mm}$ pore diameter under particle velocity of $0.32 \mathrm{~km} / \mathrm{s}$.

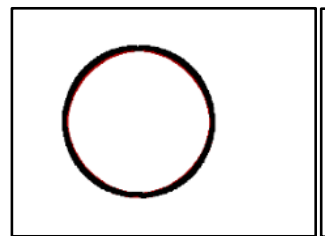

i) $t=0 \mu \mathrm{s}$

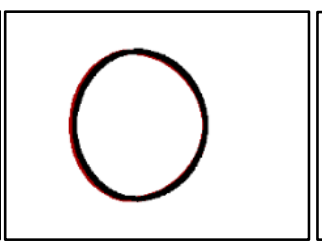

ii) $t=1.093 \mu \mathrm{s}$

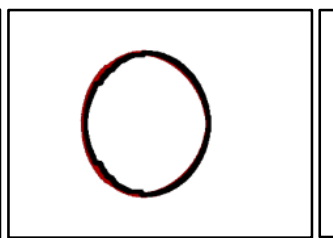

iii) $t=1.621 \mu \mathrm{s}$

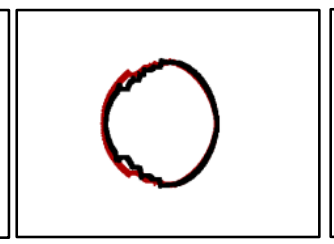

iv) $t=2.677 \mu \mathrm{s}$

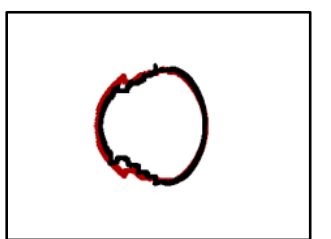

v) $t=3.205 \mu \mathrm{s}$

(b) Comparison of pore deformation obtained from the experiment and 2D simulation.

Experimental pore shapes are shown by the black curves and the red curves show the $2 \mathrm{D}$ simulation results.

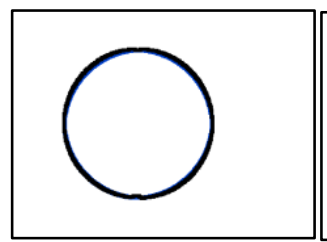

i) $t=0 \mu \mathrm{s}$

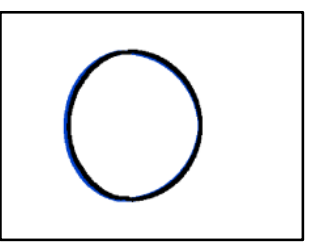

ii) $t=1.093 \mu \mathrm{s}$

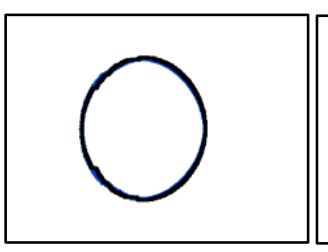

iii) $t=1.621 \mu \mathrm{s}$

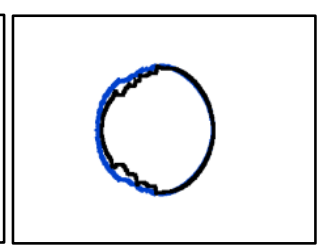

iv) $t=2.677 \mu \mathrm{s}$

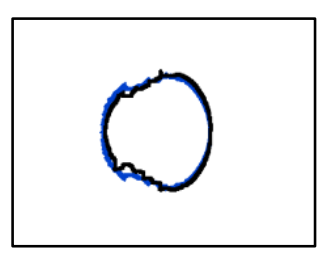

v) $t=3.205 \mu \mathrm{s}$

(c) Comparison of pore deformation obtained from the experimental and 3D simulation. Experimental pore profiles are shown by the black curves and the blue curves show the 3D simulation results.

Figure 4: Comparison of experimental and simulation results (2D and 3D for the collapse of a pore of diameter $6 \mathrm{~mm}$ in PMMA matrix impacted by a shock with particle velocity of 0.32 $\mathrm{km} / \mathrm{s}$. 


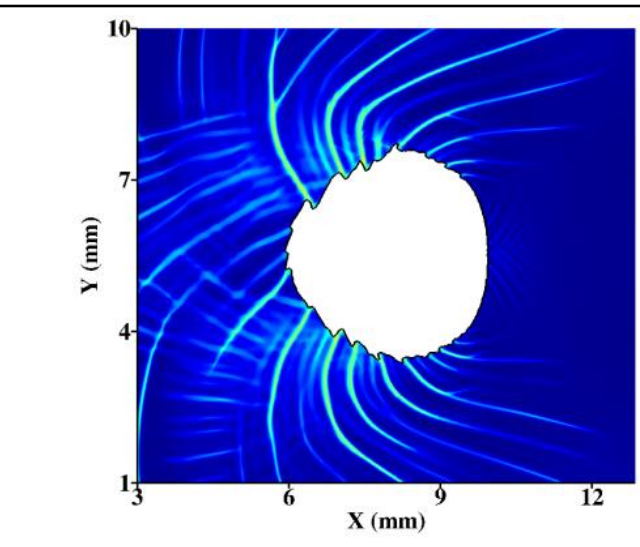

(a) $t=3.583 \mu \mathrm{s}$

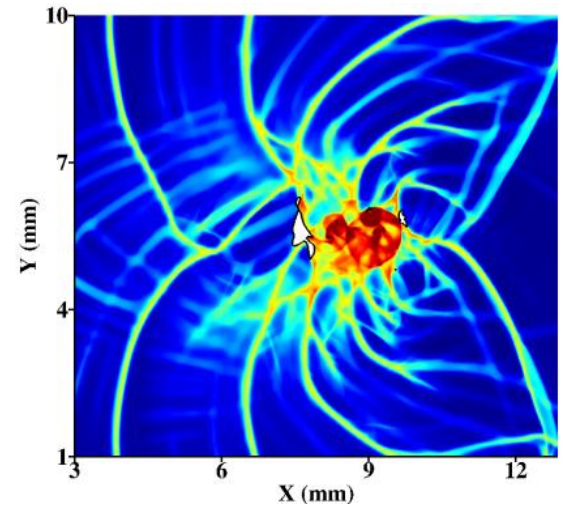

(c) $t=6.995 \mu \mathrm{s}$

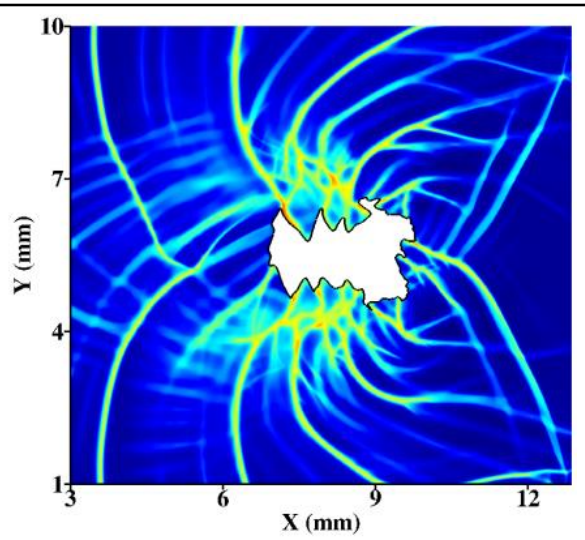

(b) $t=5.861 \mu \mathrm{s}$

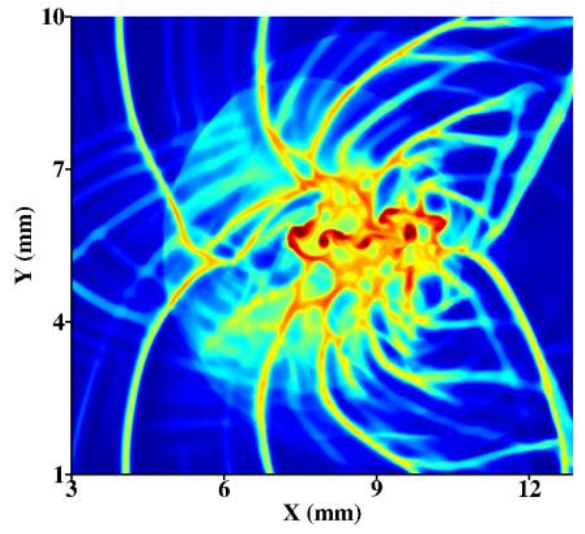

(d) $t=7.977 \mu \mathrm{s}$
Temperature (K)

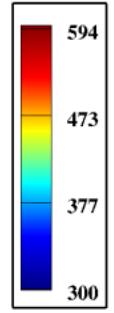

Figure 5: Temperature (K) evolution following the collapse of the pore of diameter $6 \mathrm{~mm}$ under particle velocity of $0.32 \mathrm{~km} / \mathrm{s}$. 


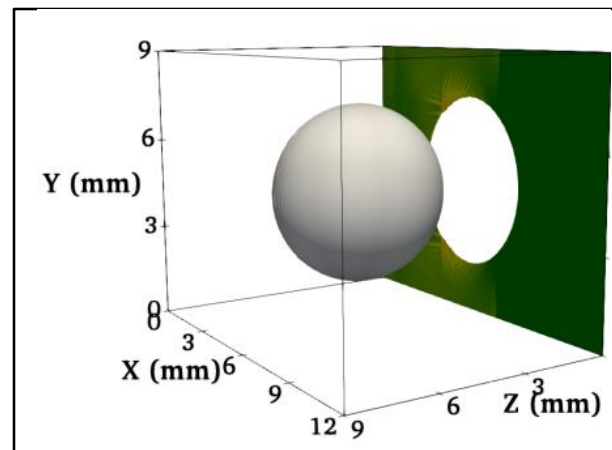

a) $t=1.7 \mu \mathrm{s}$

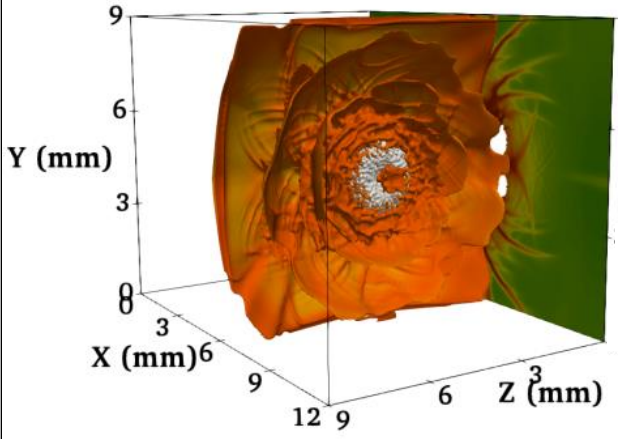

c) $t=4.976 \mu \mathrm{s}$

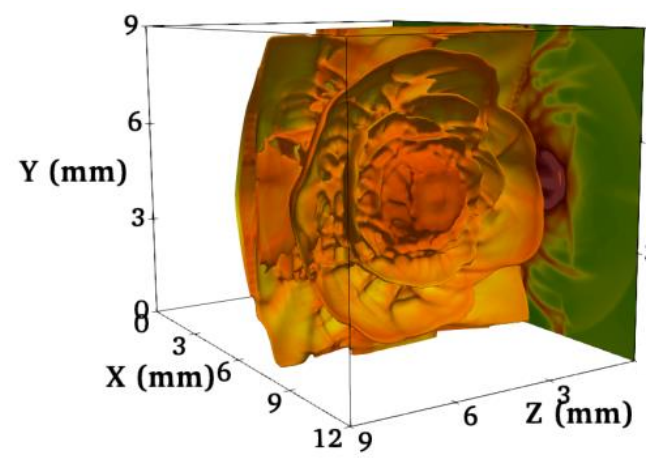

e) $t=6.609 \mu \mathrm{s}$

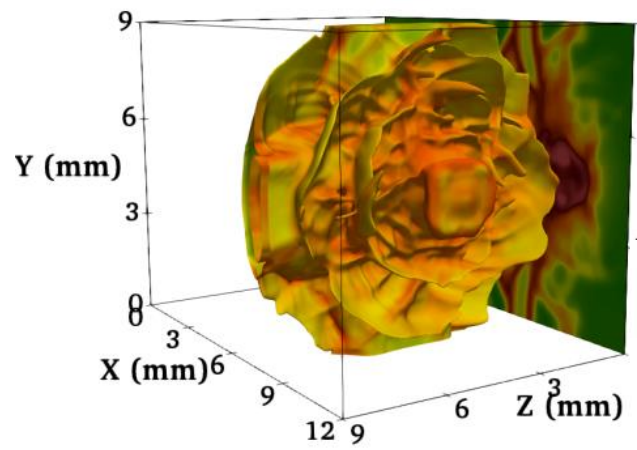

g) $t=11.848 \mu \mathrm{s}$

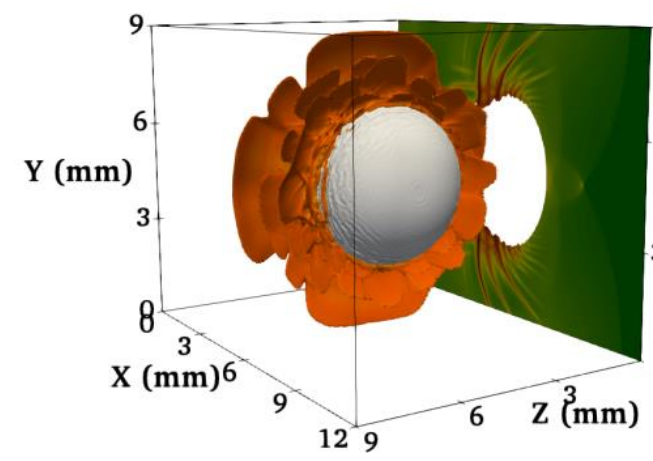

b) $t=3.193 \mu \mathrm{s}$

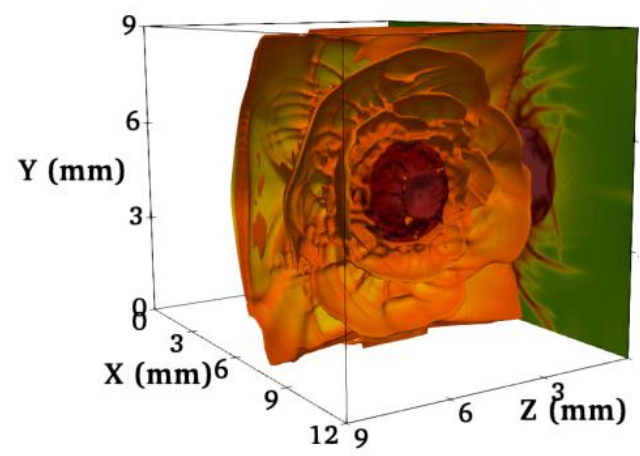

d) $t=5.747 \mu \mathrm{s}$

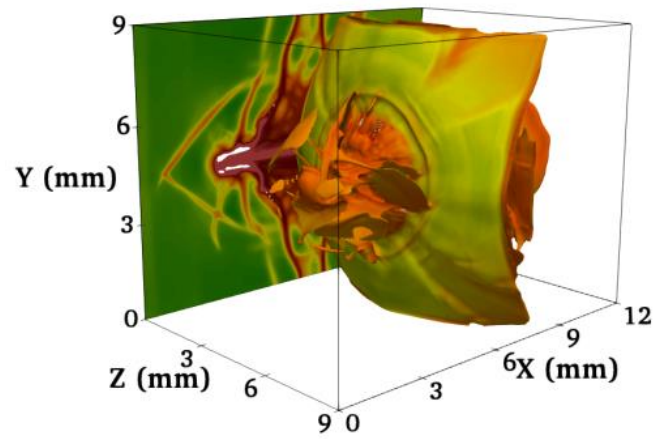

f) $t=6.609 \mu \mathrm{s}$

Temperature (K)

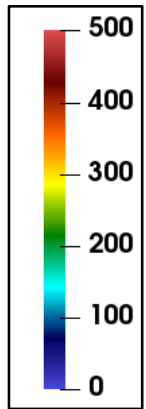

Figure 6: Comparison of experimental and simulation results for the collapse of a spherical pore of diameter $6 \mathrm{~mm}$ in PMMA impacted by a shock with particle velocity of $0.32 \mathrm{~km} / \mathrm{s}$. Isosurface of effective plastic strain colored with temperature contours $(\mathrm{K})$ are shown to visualize the adiabatic shear bands in 3D. 


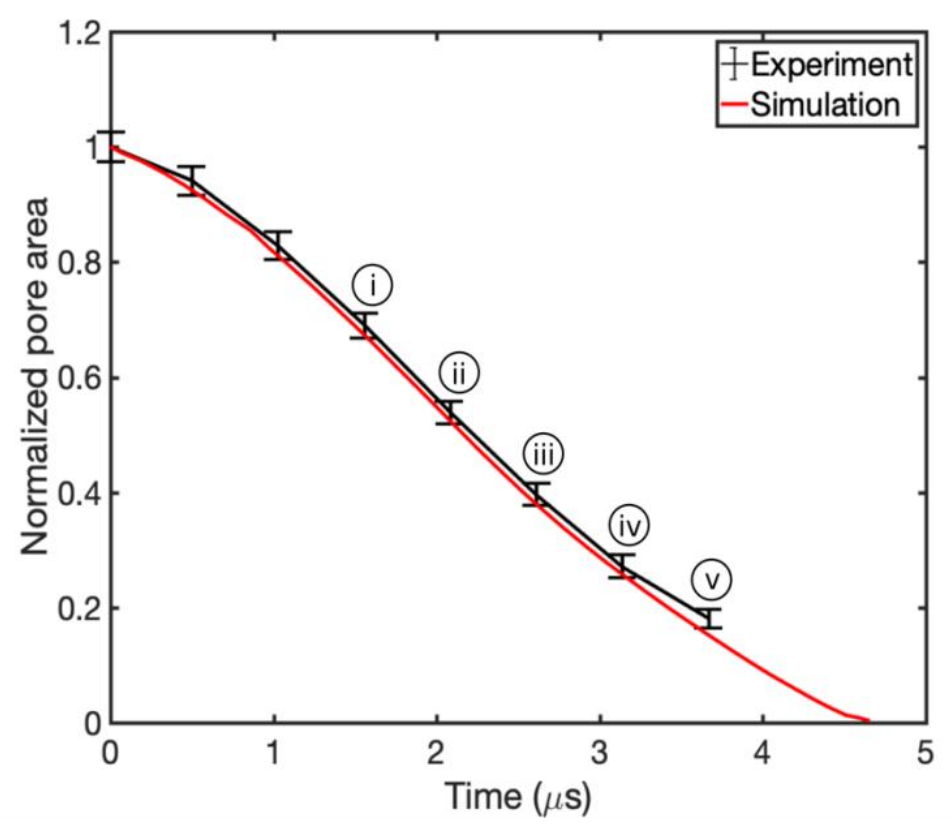

(a) Experimental and simulation results comparison of the time evolution of the normalized pore area for $6 \mathrm{~mm}$ pore diameter under particle velocity of $0.47 \mathrm{~km} / \mathrm{s}$.

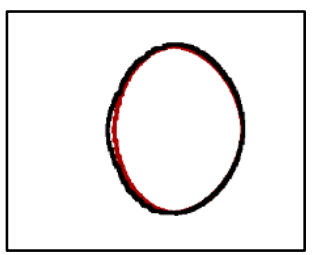

i) $t=1.587 \mu \mathrm{s}$

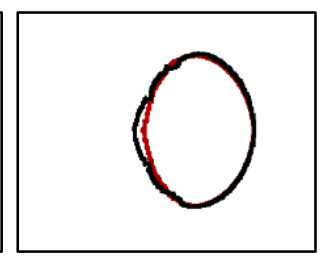

ii) $t=2.115 \mu \mathrm{s}$

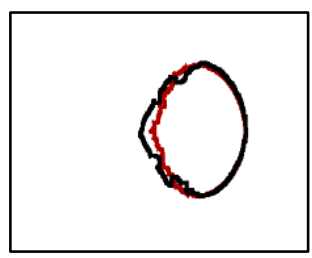

iii) $t=2.643 \mu \mathrm{s}$

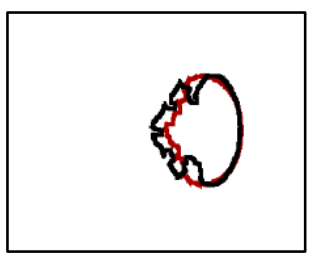

iv) $t=3.171 \mu \mathrm{s}$

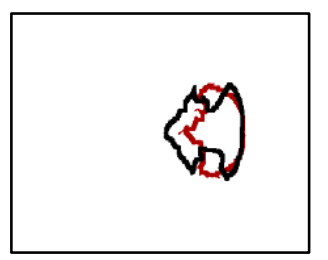

v) $t=3.699 \mu \mathrm{s}$

(b) Comparison of pore deformation profile obtained from the experimental and simulation analysis. Experimental pore profiles are shown by the black curve and the red curves show the $2 \mathrm{D}$ simulation results.

Figure 7: Comparison of experimental and simulation results for the collapse of a cylindrical pore of diameter $6 \mathrm{~mm}$ in PMMA matrix impacted by a shock with particle velocity of 0.47 $\mathrm{km} / \mathrm{s}$. 


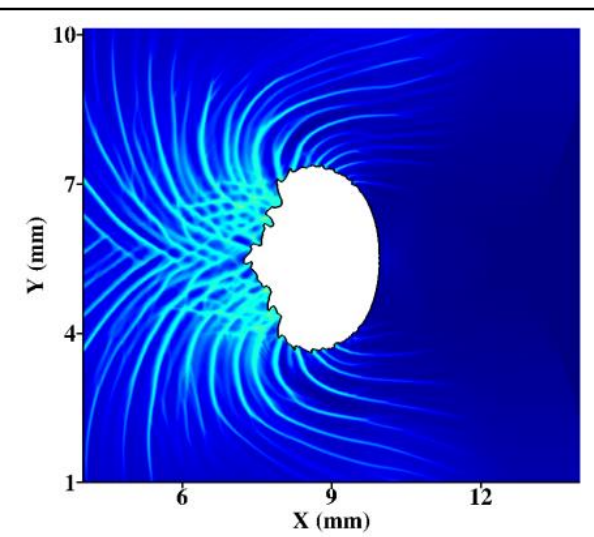

a) $t=3.161 \mu \mathrm{s}$

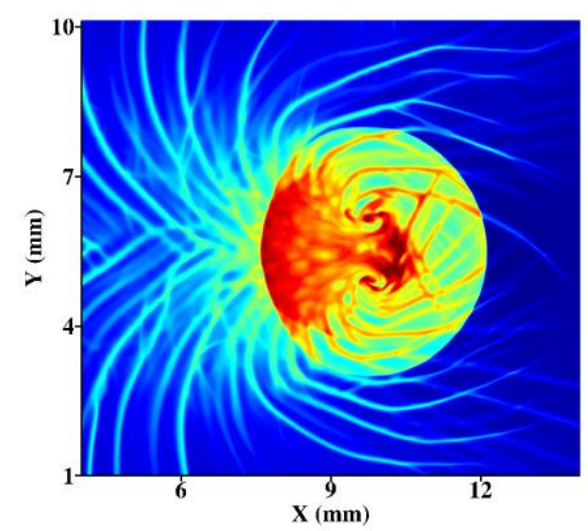

c) $t=5.227 \mu \mathrm{s}$

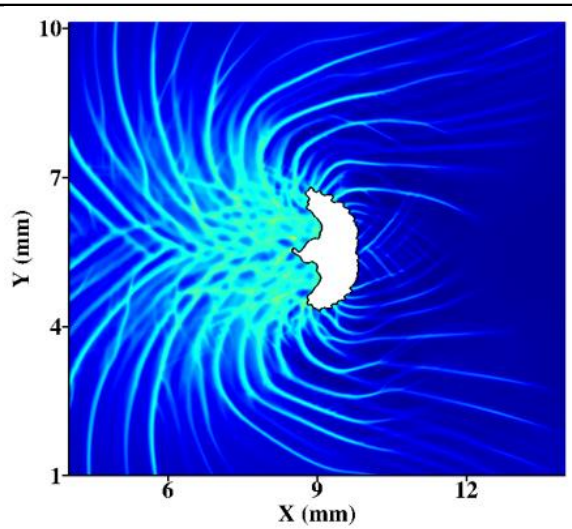

b) $t=4.154 \mu \mathrm{s}$

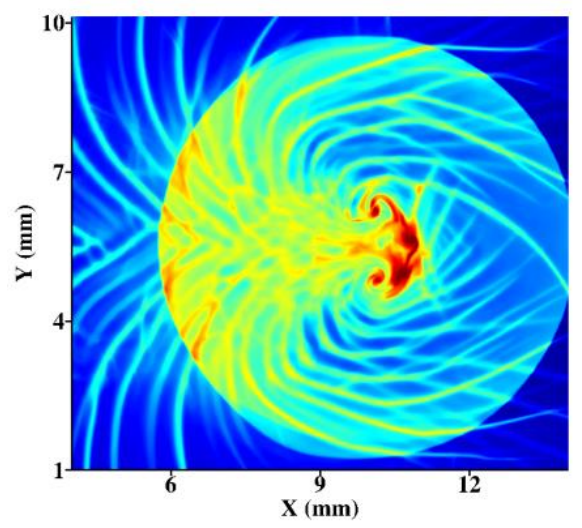

d) $t=5.78 \mu \mathrm{s}$
Temperature (K)

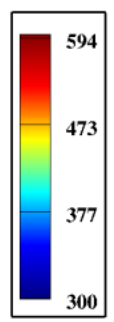

Figure 8: Temperature (K) evolution following the collapse of the pore of diameter $6 \mathrm{~mm}$ under particle velocity of $0.47 \mathrm{~km} / \mathrm{s}$. 


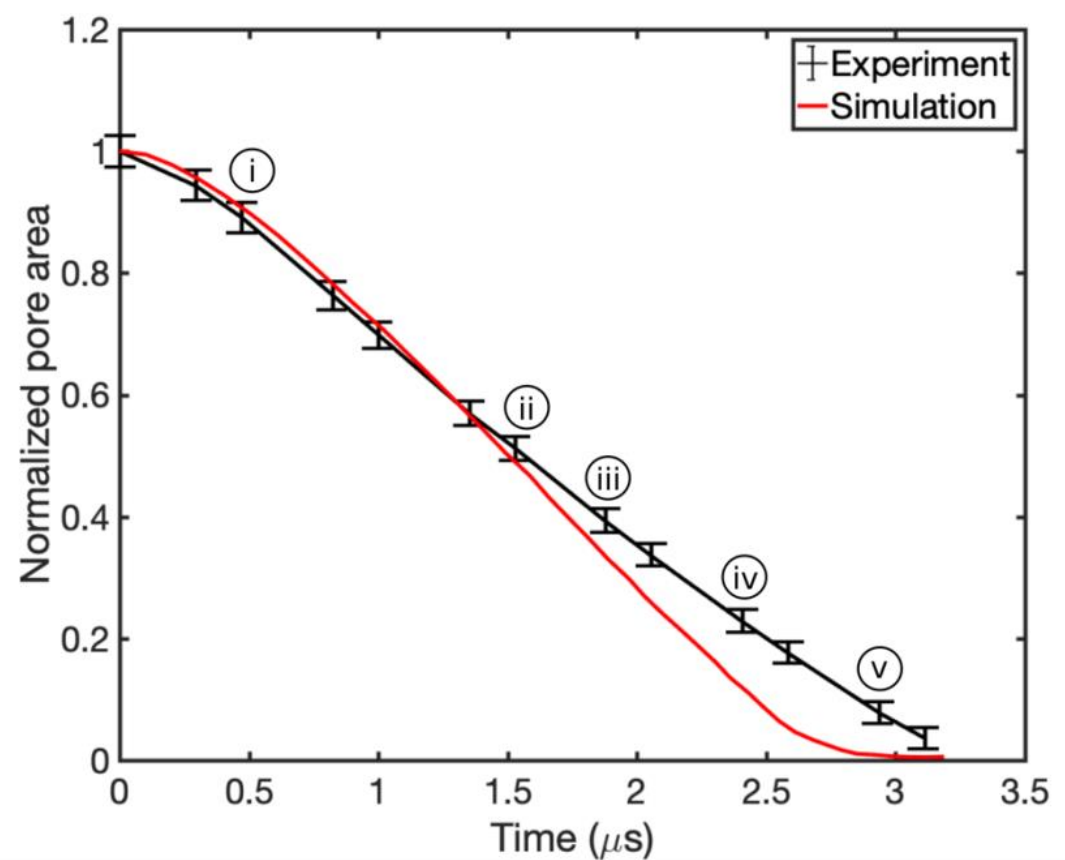

(a) Experimental and simulation results comparison of the time evolution of the normalized pore area for $6 \mathrm{~mm}$ pore diameter under particle velocity of $0.84 \mathrm{~km} / \mathrm{s}$.
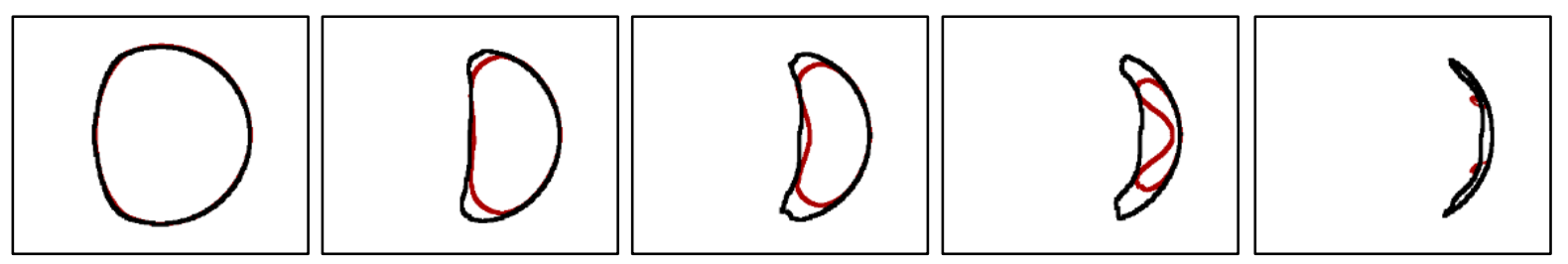

i) $t=0.485 \mu \mathrm{s}$

ii) $t=1.541 \mu \mathrm{s}$

iii) $t=1.893 \mu \mathrm{s}$

iv) $t=2.488 \mu \mathrm{s}$

v) $t=2.949 \mu \mathrm{s}$

(b) Comparison of pore deformation profiles obtained from the experimental and simulation analysis. Experimental pore profiles are shown by the black curves and the red curves show the $2 \mathrm{D}$ simulation results.

Figure 9: Comparison of experimental and simulation results for the collapse of a cylindrical pore of diameter $6 \mathrm{~mm}$ in PMMA matrix impacted by a shock with particle velocity of 0.84 $\mathrm{km} / \mathrm{s}$. 


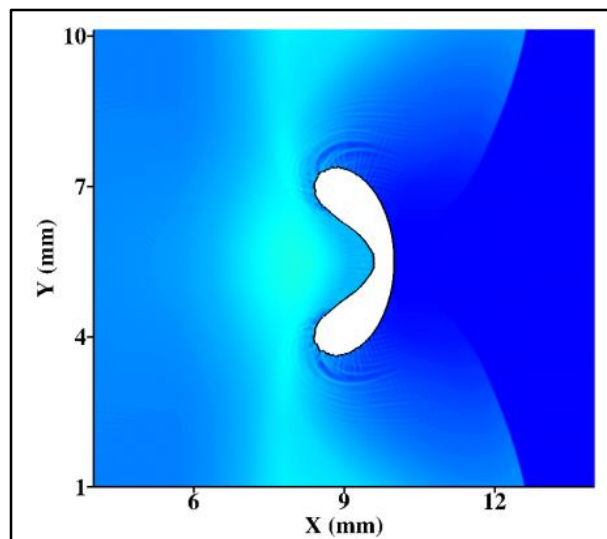

(a) $t=2.43 \mu \mathrm{s}$

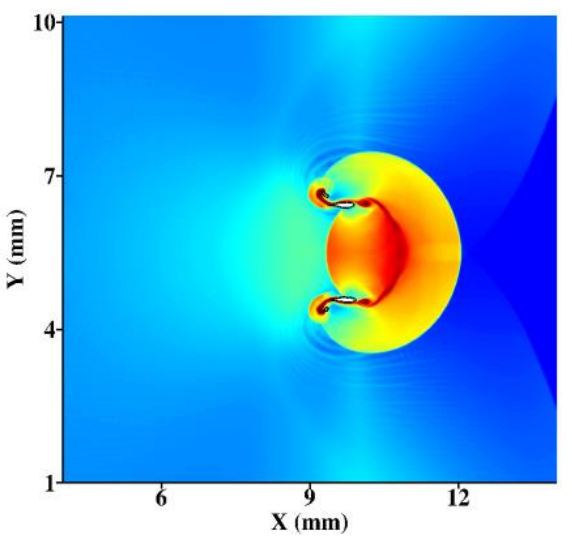

(c) $t=2.985 \mu \mathrm{s}$

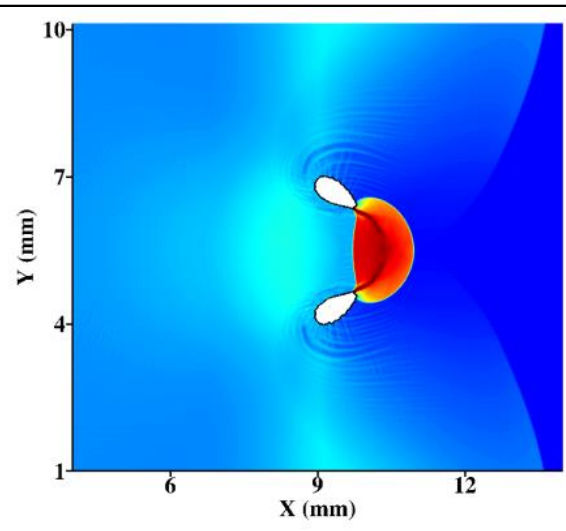

(b) $t=2.743 \mu \mathrm{s}$

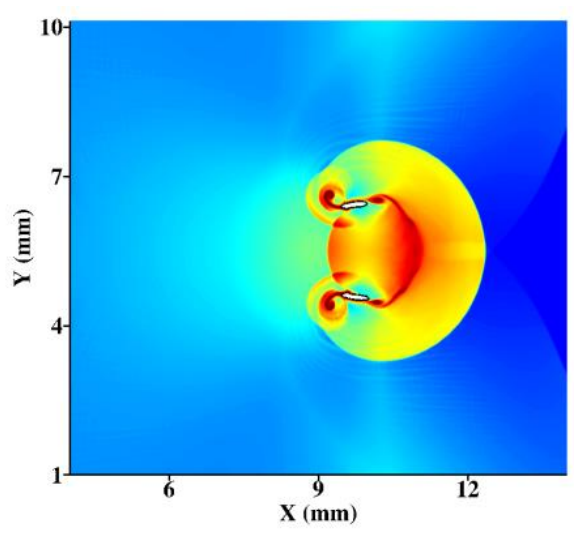

(d) $t=3.05 \mu \mathrm{s}$
Temperature (K)
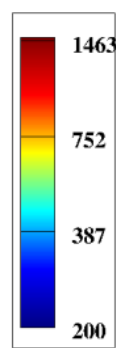

Figure 10: Temperature $(\mathrm{K})$ evolution following the collapse of the pore of diameter $6 \mathrm{~mm}$ under particle velocity of $0.84 \mathrm{~km} / \mathrm{s}$. 


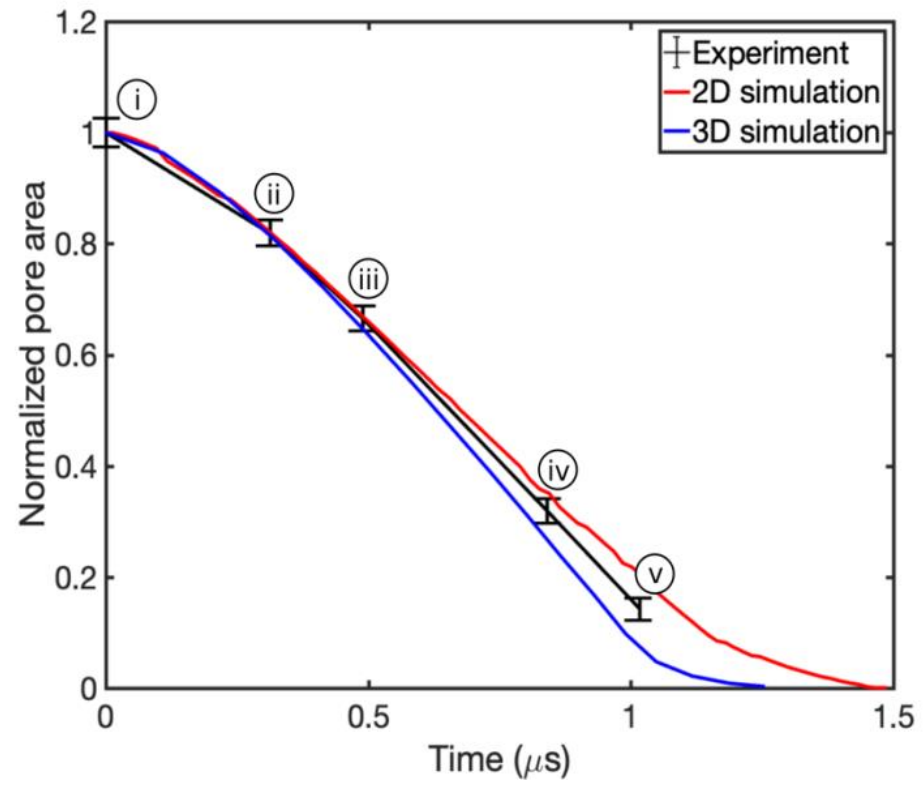

(a) Experimental and simulation results comparison of the time evolution of the normalized pore area for $6 \mathrm{~mm}$ pore diameter under particle velocity of $2.30 \mathrm{~km} / \mathrm{s}$.

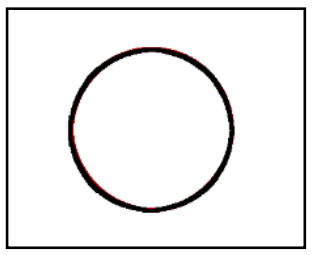

i) $t=0.0 \mu \mathrm{s}$

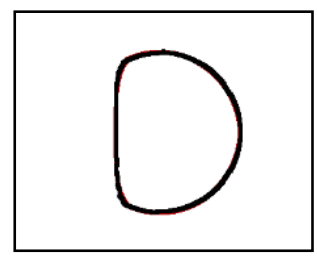

ii) $t=0.312 \mu \mathrm{s}$

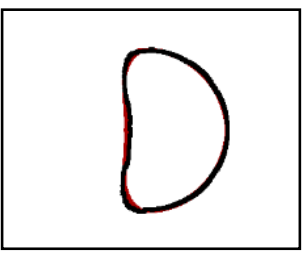

iii) $t=0.488 \mu \mathrm{s}$

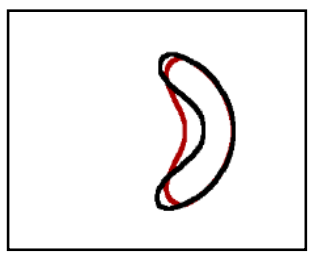

iv) $t=0.840 \mu \mathrm{s}$

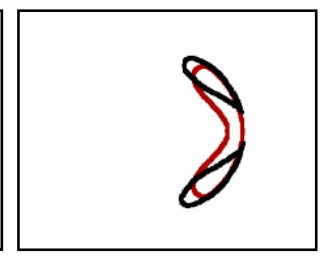

v) $t=1.016 \mu \mathrm{s}$

(b) Comparison of pore deformation profiles obtained from the experimental and 2D simulation. Experimental pore profiles are shown by the black curves and the red curves show the $2 \mathrm{D}$ simulation results.
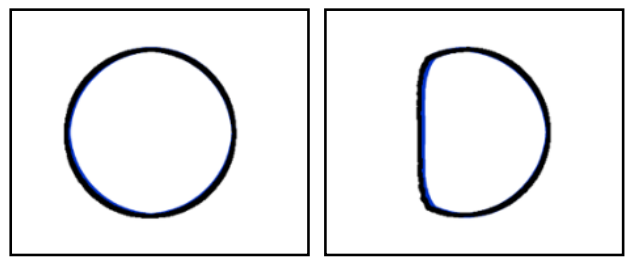

i) $t=0.0 \mu \mathrm{s}$

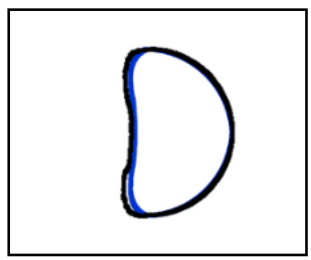

iii) $t=0.488 \mu \mathrm{s}$

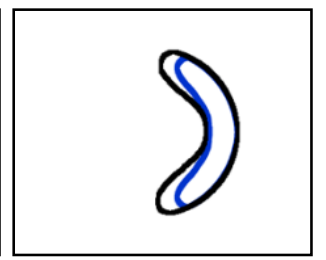

iv) $t=0.840 \mu \mathrm{s}$

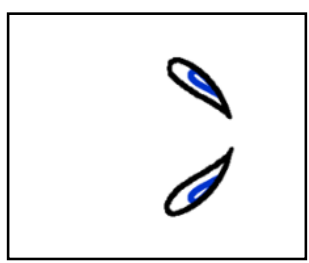

v) $t=1.016 \mu \mathrm{s}$

(c) Comparison of pore deformation profiles obtained from the experimental and 3D simulation. Experimental pore profiles are shown by the black curve and the blue curve shows the 3D simulation results.

Figure 11: Comparison of experimental and simulation results for the collapse of a pore of diameter $6 \mathrm{~mm}$ in PMMA matrix impacted by a shock with particle velocity of $2.30 \mathrm{~km} / \mathrm{s}$. 


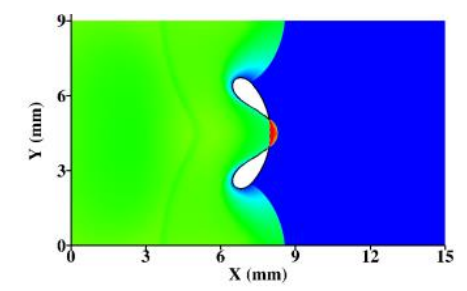

i) $t=1.51 \mu \mathrm{s}$

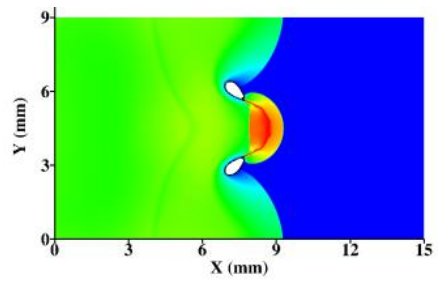

ii) $t=1.665 \mu \mathrm{s}$

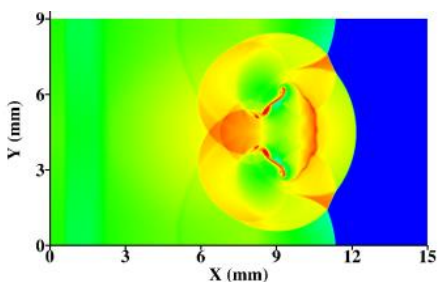

iii) $t=2.13 \mu \mathrm{s}$

(a) Temperature $(\mathrm{K})$ contours from the collapse of a circular pore of diameter $6 \mathrm{~mm}$

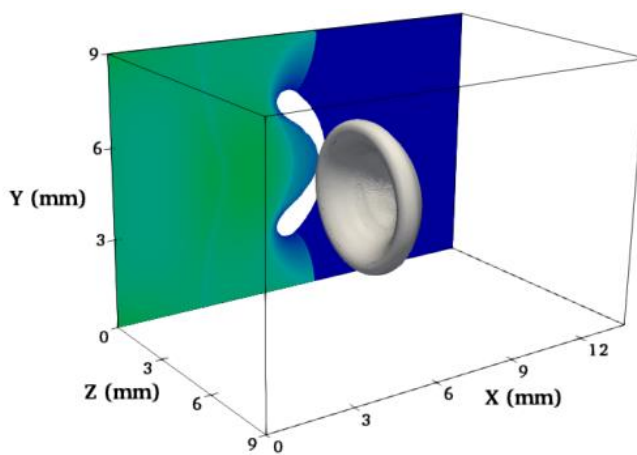

i) $t=0.99 \mu \mathrm{s}$

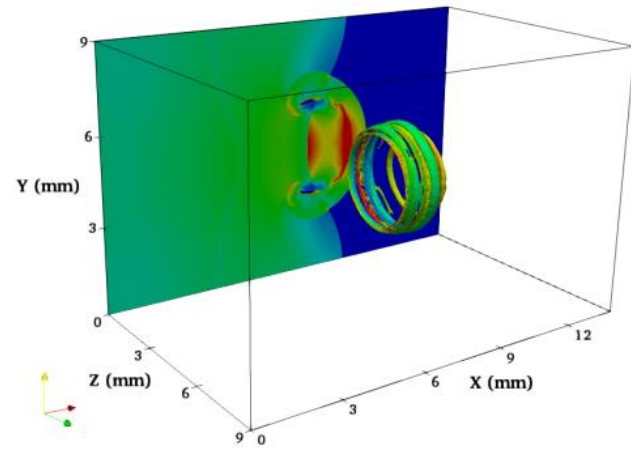

iii) $t=1.32 \mu \mathrm{s}$

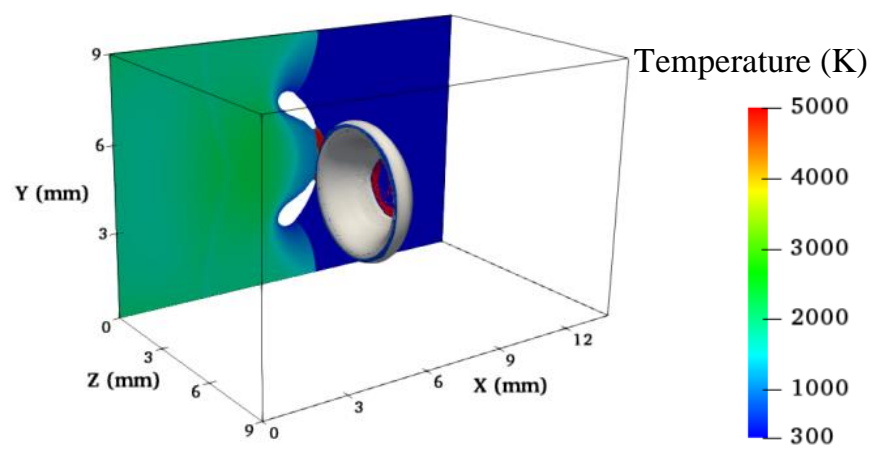

ii) $t=1.049 \mu \mathrm{s}$

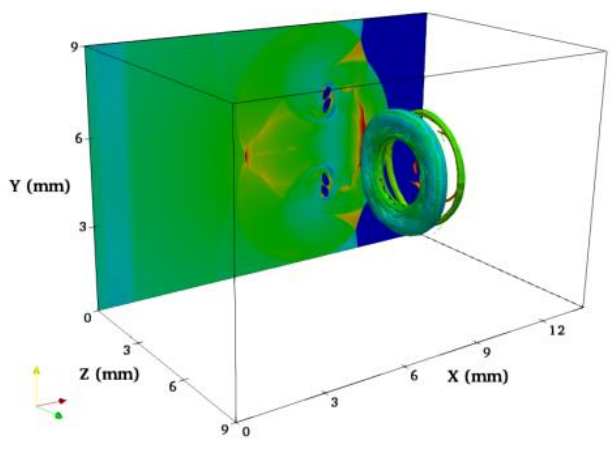

iv) $t=1.638 \mu \mathrm{s}$

(b) Iso-surface of $\lambda_{2}$ (for vorticity) colored with temperature for the collapse of the spherical pore

Figure 12: Temperature $(\mathrm{K})$ evolution following the collapse of the circular and spherical pore of diameter $6 \mathrm{~mm}$ under particle velocity of $2.30 \mathrm{~km} / \mathrm{s}$. 


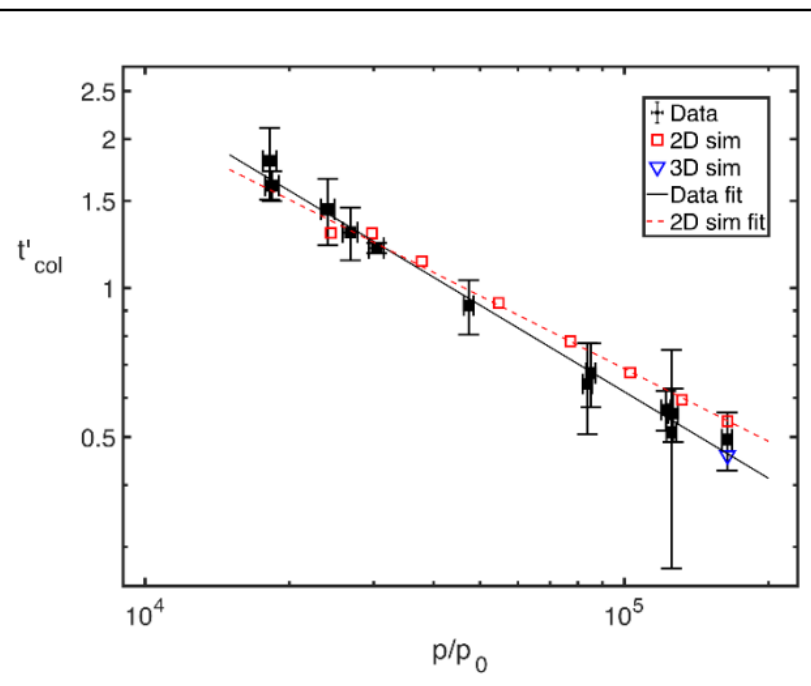

(a) Variation of time to collapse with loading pressure

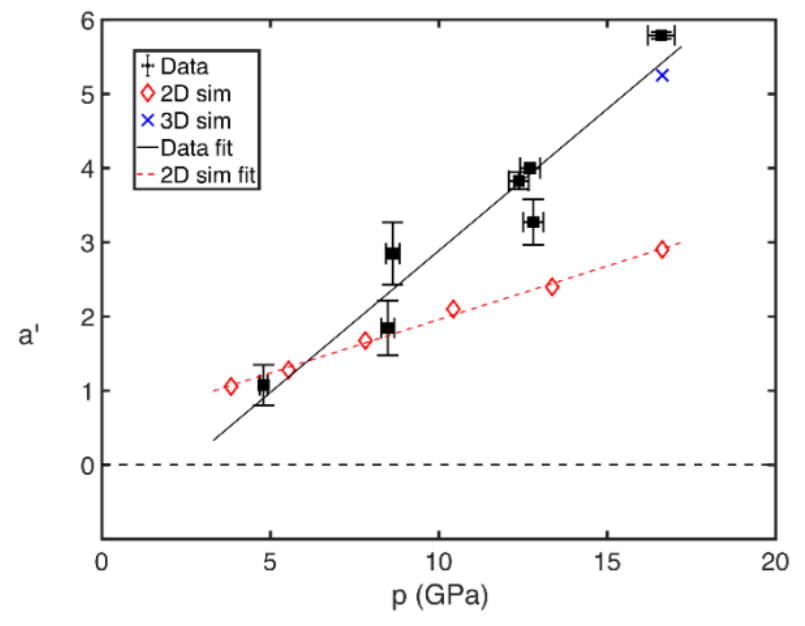

(b) Variation of time to centerline pore acceleration (jet acceleration) with loading pressure

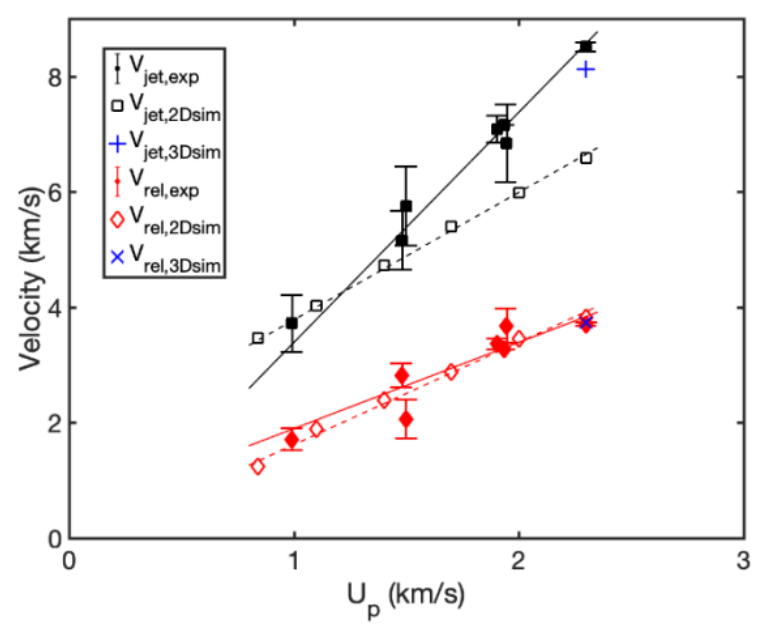

(c) Variation of jet and release velocities with applied particle velocity

Figure 13: Comparison of variation of time to collapse, jet acceleration and jet velocity with respect to the loading strength between experiment and simulation results. 